\title{
IL CARTEGGIO TRA GAETANO CATTANEO, FREDERIK MÜNTER E PIETRO TAMBURINI. FRA NUMISMATICA E GIANSENISMO
}

\author{
FEDERICO ZULIANI (*) \\ Nota presentata dal m.e. Enrico I. Rambaldi \\ (Adunanza del 14 gennaio 2014)
}

SunTO. - La nota esamina il carteggio tra Gaetano Cattaneo e il dotto danese Frederik Münter di cui sono sopravvissute 12 lettere (datate 1815-1825) oggi custodite presso la Biblioteca Reale di Copenaghen. Nato da ragioni prettamente numismatiche, lo scambio epistolare consente purtuttavia di ricostruire una parte significativa dei rapporti di Münter con l'Italia prima e dopo il suo rientro in patria nel 1787 al termine di un quadriennio trascorso tra Napoli e Roma. Da una parte offre diverso materiale per tratteggiare meglio i legami di Münter con la Lombardia dove risiedette alcune settimane sulla via del ritorno in Danimarca, frequentando gli ambienti latomisti, e dove si fece amico, tra gli altri, di Luigi Bossi, Gian Rinaldo Carli, Carlo Innocenzo Isimbardi e del conte Wilzeck. Dall'altra, il carteggio ci informa nel dettaglio della profonda stima che unì Münter ai teologi giansenisti dell'Università di Pavia; Pietro Tamburini e Giuseppe Zola in primis. La nota illustra infine come Münter, prossimo alle istanze storico-teologiche dei prelati giansenisti italiani, si impegnò a farne conoscere il pensiero al pubblico colto dell'Europa settentrionale.

$$
* * *
$$

ABSTRACT. - Cet essai examine la correspondance établie entre Gaetano Cattaneo et le savant danois Frederik Münter, dont douze lettres, datées des années 1815-1825, ont été conservées à la Bibliothèque Royale de Copenhague. Commencé pour des raisons strictement numismatiques, l'échange épistolaire permet toutefois de reconstruire une partie significative des rapports que Münter a entretenus avec l'Italie avant et après son retour dans sa patrie, survenu en 1787 au terme de quatre ans passés entre Naples et Rome. Cette correspondance apporte d'un côté diverses informations permettant de

(*) The Warburg Institute, School of Advanced Study, University of London, UK. E-mail: federico.zuliani@gmail.com 
mieux retracer les liens de Münter avec la Lombardie où il résida quelques semaines lors du retour en Danemark, et où il fréquenta les milieux latomistes et se lia d'amitié avec entre autres Luigi Bossi, Gian Rinaldo Carli, Carlo Innocenzo Isimbardi et le comte Wilzeck. D'un autre côté, elle nous renseigne en détail sur la profonde estime unissant Münter aux théologiens jansénistes de l'Université de Pavie, Pietro Tamburini et Giuseppe Zola en premier lieu. Enfin, cet essai illustre comment Münter, proche des conceptions historico-théologiques des prélats jansénistes italiens, s'efforça d'en faire connaître la pensée au public cultivé de l'Europe du Nord.

$* * *$

Il contributo di Federico Zuliani muove dall'esame delle lettere che il numismatico milanese Gaetano Cattaneo (1771-1841) scrisse tra il 1815 e il 1825 a Frederik. Münter (1761-1830), teologo, orientalista, numismatico, storiografo e massone. L'amicizia epistolare tra $i$ due studiosi, propiziata da conoscenze comuni, s'interruppe per il risentimento di Münter, contrariato che Cattaneo avesse omesso di compiere per lui alcune ricerche nell'archivio di Mantova.

Münter è figura interessante nel panorama culturale e politico italiano del decennio che precede la Rivoluzione francese, $i$ successivi sussulti repubblicani italiani, il periodo napoleonico e la Restaurazione. Nato in Germania ma trasferito in Danimarca ancora bambino, quando il padre Balthasar venne chiamato come pastore alla Sankt Petri Kirke, nei Wander und Lehrjahre giovanili Münter, poi professore di teologia e rettore dell'Università della capitale danese, ebbe intensi contatti con logge massoniche europee. Nel 1782 frequentò in Germania gli Illuminati di Baviera, corrente massonica razionalistica che esercitò grande influenza negli ambienti riformatori europei. Della successiva Studienreise intrapresa da Münter in Italia s'occupò nel 1938 ne «La Critica» Benedetto Croce, con uno studio poi raccolto negli Aneddoti di varia letteratura (vol. III, pp. 168-180) e intitolato Federico Münter e la massoneria di Napoli nel 1785-86. «Ottenuto dal governo di Copenaghen, sul fondo ad usus publicos, uno stipendio per un viaggio d'istruzione» (p. 170), scrive Croce, dal 1784 al 1787 Münter fu in Italia «come un agente o (si direbbe nel linguaggio politico odierno) un organo di collegamento della massoneria europea» ( $p$. 169), in particolar modo degli Illuminati. Di Münter Croce studiò $i$ diversi soggiorni napoletani, durante i quali strinse rapporti «con rappresentanti dell'illuminismo napoletano» ( $p$. 168) quali Gaetano Filangieri (1752-1788), Pasquale Baffi (1749-1799) e Mario Pagano (1748-1799), e con gentildonne come Eleonora Fonseca Pimentel (1752-1799). Una decina di anni dopo, nel 1796, quando la sorella di Münter, Friederike Brun, giungerà a Napoli e cercherà gli amici del fratello, troverà ch'erano «quasi tutti nelle carceri o dispersi dagli esilì» (p. 168). Nel 1799, con la sconfitta della rivoluzione repubblicana, «su tutti quegli [spiriti] nobilissimi» (p. 179) s'abbatté la repressione, e più d'uno degli antichi interlocutori di Münter, come il Baffi, il Pagano e la Fonseca Pimentel, sali sul patibolo.

Pur caratterizzate soprattutto dall'erudizione numismatica, nelle lettere che Cattaneo scrisse a Münter quando alle istituzioni prima repubblicane e poi napoleoniche era ormai succeduta la Restaurazione si avvertono nitidi gli echi di quegli anni ribollenti. E se i rapporti di Münter con l'Italia centro-meridionale risultano già ben studiati, lo studio di Zuliani ha il merito di avvalersi delle lettere di Cattaneo per esplorare quelli con l'Italia settentrionale, in particolare con la Lombardia, dove Münter, ormai sulla via del ritorno in Danimarca, sostò alcune settimane nel 1787. Dalle lettere risulta infatti che pur se politicamente e culturalmente assai meno rilevanti che non quelle napoletane, anche le relazioni che Münter allacciò con personalità lombarde furono segnate dagli eventi rivoluzionari. 
Del più ampio panorama tracciato da Zuliani, mi restringo qui a dire brevemente di quelle, tra le personalità presenti o nominate nel carteggio, che furono membri del nostro Istituto. La prima è lo stesso Gaetano Cattaneo, che nel 1787, quando Münter soggiornò a Milano, aveva solo sedici anni e non lo conobbe personalmente. M.E. del nostro Istituto, Gaetano Cattaneo fu personalità non di primo piano. Pittore di talento, ai tempi della Casa d'Austria entrò come giovane disegnatore nella zecca, dove negli anni dell' influenza francese divenne, grazie all'appoggio di Francesco Melzi d'Eril, impiegato. Quando, dopo la sconfitta subita nel 1813 a Lipsia, Napoleone pretese che le monete e le medaglie auree di proprietà delle zecca venissero fuse per dare oro al governo di Parigi, Cattaneo, che nel frattempo era divenuto esperto di numismatica, con la copertura politica di Giuseppe Prina si adoprò per salvarle. Cominciò cosi la carriera di numismatico "istituzionale", divenendo dapprima custode delle monete e medaglie di pregio della zecca, poi conservatore del Gabinetto numismatico di Brera, e infine, sotto la Restaurazione, direttore dello stesso. Culturalmente vicino a Carlo Porta, a Giuseppe Bossi, a Tommaso Grossi e ad Alessandro Manzoni, fu autore di rare pubblicazioni, tra le quali mette conto ricordare il Catalogus populorum, urbium et regum quorum nummi adservantur in Regio Officinae monetariae Museo Mediolanensi $e$ il discorso funebre per Giuseppe Bossi, nel quale, seppur cautamente, lodò l'opera culturale dell'ormai defunto Regno d'Italia, ricordando ch'era stato «col favor del governo [che Giuseppe Bossi aveva potuto aprire] una scuola [di pittura] in sua casa» (Discorso recitato dal Signor Gaetano Cattaneo, Conservatore del R.I. Gabinetto delle Medaglie, nel funerale del Cavaliere Giuseppe Bossi il giorno 11 dicembre 1815, Milano, presso Federico Agnelli, s.d., p. 7).

Di ben altro spicco la personalità di Luigi Bossi (1758-1835), che Münter aveva conosciuto nel 1787. Membro Pensionato del nostro Istituto, nel quale entrò nel 1803, prima di spogliarsi dell'abito talare fu molto vicino a Filippo Visconti, l'arcivescovo di simpatie gianseniste che Giuseppe II aveva insediato a Milano. Quando Münter lo conobbe Luigi Bossi era un autore affermato, che aveva dibattuto il problema delle alienazioni ecclesiastiche in una Lettre a M. d'Alembert (1781) e pubblicato in forma anonima Del cattolicesimo della Chiesa di Utrecht (1786), opera d'ispirazione giansenista che ebbe notevole risonanza e venne tradotta in francese (1788). In quegli anni Bossi fu molto vicino alla loggia milanese della Concordia, ispirata alle idee razionalistiche degli Illuminati e retta da Johann Joseph $v$. Wilzeck, succeduto nel 1782 come plenipotenziario al Firmian. Luigi Bossi ebbe dipoi vita tribolata: per sottrarsi alle conseguenze di un fallimento riparò a Venezia, dove si schierò con $i$ sostenitori di Napoleone e fu probabilmente il compilatore della lista di manoscritti ed opere d'arte, dalla quale Bonaparte avrebbe attinto le opere da portare a Parigi. Cosi fu per favore di Napoleone che nel 1798 Bossi tornò a Milano ed entrò nel Gran Consiglio della Repubblica. Nominato rappresentante della Repubblica cisalpina presso la Repubblica ligure e la corte sabauda di Torino, grazie all'amicizia con Melzi d'Eril dopo la battaglia di Marengo (1800) venne nominato prefetto alle biblioteche e agli archivi. Caduto Napoleone, per le condizioni economiche molto critiche si ridusse a vivere di una modesta pensione e della sua penna, pubblicando opere voluminose ma compilative, come $i 19$ voll. Della istoria d'Italia antica e moderna, apparsa a Milano tra il 1819 e il 1823. A Münter, che gli chiedeva notizie dell'amico conosciuto negli ambienti massonici ai tempi del suo splendore, Cattaneo rispose «Monsignor Bossi non è più né Monsignore né prete. All'epoca della nostra politica pazzia egli abbandonò lo Stato ecclesiastico e gettossi nel vortice degli affari, a tal che fu del Corpo Legislativo, Prefetto delle Biblioteche degli Archivi, ed infine Agente diplomatico. Ora egli non è più nulla di tutto ciò, e vive ritiratissimo, e ruinato».

Un altro nostro accademico nominato nelle lettere è Carlo Innocenzo Isimbardi 
(1767-1824), la cui madre, Maddalena, era sorella di Cesare Beccaria. Pensionato del nostro Istituto, direttore generale dell' Amministrazione delle Monete e responsabile delle miniere, il 5 maggio 1814 Isimbardi presentò nel nostro Istituto una memoria «Sui piccoli forni di fusione a manica portatili».

Zuliani informa che dal carteggio di Münter con uno studioso tedesco si evince ch'ebbe rapporti anche con Carlo Ottavio Castiglioni (1785-1849), che del nostro Istituto fu Presidente. I due non si conobbero personalmente, dato che quando Münter soggiornò a Milano e Pavia Castiglioni aveva appena due anni. L'interesse di Münter per lui fu dunque dovuto a motivi non "politici" né "di massoneria", ma solo scientifici, certo originati dall'imponente monografia sulle monete cufiche del Gabinetto numismatico di Brera che Castiglioni pubblicò nel 1819.

Ma l'amico "italiano" di maggior spicco del carteggio è Pietro Tamburini (17371827). Membro Onorario del nostro Istituto, nel 1779 Tamburini fu nominato da Maria Teresa titolare di Teologia morale nell'Ateneo pavese, del quale fu anche rettore. Teologo giansenista, nel 1786 fu con Scipione de' Ricci (1740-1810), vescovo di Prato e Pistoia, il principale animatore del Sinodo diocesano di Pistoia, che ambi a promuovere una riforma giansenistica della Chiesa. Allontanato nel 1794 dall'università, vi venne reinsediato dalla Repubblica cisalpina come titolare di Filosofia morale e Diritto naturale. Rettore del liceo di Brescia quando la città cacciò la Serenissima, nel 1799 si ritirò in campagna, ma venne nuovamente reinsediato nell'università di Pavia dal Regno d'Italia, dove fu confermato dagli Asburgo dopo la caduta di Napoleone e senza ch'egli avesse abiurato le sue precedenti concezioni; ce lo mostra, ad es., la sua nota Sulla vera definizione del fanatismo, apparsa nel secondo volume (1821) delle Memorie del nostro Istituto pubblicate sotto la Restaurazione, nella quale si ravvisa il persistente legame dell'ormai anziano professore e teologo con gli Idéologues. Del carteggio studiato da Zuliani Tamburini fu attore diretto, dato che che l'ormai quasi novantenne giansenista affidò a Cattaneo, perchéle trascrivesse nelle sue lettere, note nelle quali dava prova d'intatta combattività: «nella rivoluzione delle cose politiche e degli umani cervelli Iddio mi volle sul campo di battaglia», scriveva in una nota, mentre in un'altra si mostrava avverso all'ortodossia della Chiesa di Roma e si rallegrava che in Germania ci fosse un rinnovamento religioso, ed esprimeva l'augurio che si estendesse a tutta la Chiesa: «Faccia il Signore che questi germi coltivati a dovere portino il frutto desiderato in tutta la Chiesa Cattolica, come fa sperare il movimento che prendono nell'Alemagna le cose ecclesiastiche».

Con l'esame e l'interpretazione di due lettere inedite indirizzate a Münter dal giansenista pavese Giuseppe Zola (1739-1806), professore di storia ecclesiastica prima e di storia antica poi presso l'università e il seminario di Pavia e molto legato a Tamburini, Zuliani mostra infine che il rapporto di Münter con Tamburini nasceva proprio dal vivo interesse dello studioso danese per le correnti gianseniste italiane.

(Enrico I. Rambaldi)

\section{INTRODUZIONE A UN CARTEGGIO A SEI MANI}

In appendice a una lettera di Gaetano Cattaneo inviata il 2 marzo 1819 e indirizzata a Luigi Rossari, all'epoca ancora studente presso l'Almo Collegio Borromeo di Pavia, è presente un breve poscritto che recita: «Ricordati de' miei complimenti all'incomparabile Tamburini a 
nome anche del Signor Münter di Coppenhagen, il quale sempre m'incarica di tale gratissima incombenza ogni volta che ha l'amicizia di scrivermi». ${ }^{1}$ Nei carteggi di Gaetano Cattaneo sino ad oggi pubblicati, come quello recentemente curato da Serena Bertolucci e da Giovanni Meda Requier, ${ }^{2}$ il nome di Münter non compare, eppure la formula impiegata in questa occasione da Cattaneo, «ogni volta che ha l'amicizia di scrivermi», testimonia una consuetudine ripetuta e un rapporto che, se non intimo, pare essere per lo meno molto cordiale, come prova d'altronde la dichiarazione di amicizia; un riferimento questo particolarmente significativo perché fatto in presenza di un altro amico, pur molto più giovane, come appunto il Rossari, almeno in potenza geloso, come lo sanno essere, forse, solo gli amici. Il poscritto sopra riportato risulta specialmente interessante soprattutto per la citazione, e l'accostamento, di due importanti personaggi della vita culturale europea a cavallo tra Diciottesimo e Diciannovesimo secolo, quali Pietro Tamburini, ${ }^{3}$ figura di spicco del giansenismo italiano, e quel «Signor Münter di Coppenhagen», che altri non è che Frederik Münter, il celebre dotto danese. ${ }^{4}$ Personalità complessa e di grande fascino, Münter fu

1 Milano, Centro Nazionale Studi Manzoniani, Codice Pozzi, 156r. La lettera è edita in Le lettere di Carlo Porta e degli amici della Cameretta. Seconda edizione accresciuta e illustrata, a cura di D. Isella, Milano e Napoli, 1989, 342. Ringrazio Jone Riva per aver portato alla mia attenzione il testo. Qui come altrove si è optato per una trascrizione diplomatica dei documenti.

2 A. Manzoni, Carteggi letterari I, a cura di S. Bertolucci e G. Meda Requier, 2 voll, Milano, 2010.

3 Al riguardo: G. Mantese, Pietro Tamburini e il giansenismo bresciano, Brescia, 1942; I. Garlaschi, Vita cristiana e rigorismo morale: studio storico-teologico su Pietro Tamburini, 1737-1827, Roma, 1985; M. Comini, Pietro Tamburini 1737-1827. Un giansenista lombardo tra Riforma e Rivoluzione, Brescia, 1992 e l'importante volume di saggi a cura dello Stesso e di D. Montanari, Pietro Tamburini e il Giansenismo Lombardo, Brescia, 1993, dove va segnalato, per quanto riguarda la vita e l'opera, specialmente l'articolo di Pietro Stella, Pietro Tamburini nel quadro del giansenismo italiano, alle pagine 151-204. Merita d'essere menzionato poi, in ambiente non italiano, il recente studio di M.W.F. Stone, The Antiquarian and The Moderniser: Giovanni Lorenzo Berti (1696-1766), Pietro Tamburini (1737-1827), and Contrasting Defenses of the Augustinian Teaching on Unbaptised Infants in Eighteenth Century Italy, Quaestio 6 (2006), 335-372, specialmente 362-372.

4 La bibliografa dedicata a Münter è vastissima, come del resto lo sono le opere di cui fu autore. Per la vita un'ottima sintesi è quella offerta da Bjørn Kornerup alla voce Friderich Münter, in Dansk biografsk leksikon, 16 voll, København, 1979-1984, X, 197-201 mentre fondamentali rimangono il primo volume, Hans Levned og Personlighed, del grande lavoro curato da A. Rasmussen e $\varnothing$. Andreasen, Frederik 
ecclesiastico, «agente massonico», ${ }^{5}$ viaggiatore indefesso e soprattutto studioso dai vasti interessi che si occupò, fra le altre cose, di scienze bibliche, di copto e di lingue orientali, di archeologia, di storia degli ordini cavallereschi e non ultimo di numismatica. Proprio gli interessi numismatici ${ }^{6}$ sono quelli a cui parrebbe più logico rivolgersi per provare a capire le ragioni della conoscenza tra Münter e Cattaneo il quale, all'epoca della lettera a Rossari, ricopriva, già da diversi anni, la carica di conservatore del Gabinetto Numismatico di Brera. ${ }^{7}$

Lo studio del carteggio superstite di Münter, oggi presso Det Kongelige Bibliotek di Copenaghen, ha fatto emergere la presenza di un piccolo fondo di dodici lettere inviate da Cattaneo a Münter tra il 1815 e il 1825 e che costituiscono la corrispondenza epistolare da cui nacque quell'amicizia a cui $\mathrm{fa}$ riferimento Cattaneo scrivendo al

Münter, et Mindeskrift, 7 voll, København, 1925-1949 che comprende anche, ai volumi II-IV, i diari di viaggio di Münter, Aus den Tagebüchern Friedrich Münters; Wander- und Lebrjabre eines dänischen Gelebrten, e, ai volumi V-VII, una selezione della corrispondenza, Aus dem Briefwechsel Friedrich Münters; Europäische Beziebungen eines dänischen Gelebrten 1780-1830. Tra i contributi più recenti meritano d'essere menzionate le monografie di E. Rosenstrauch-Königsberg, Freimaurer, Illuminat, Weltbürger. Friedrich Münters Reisen und Briefe in ibren europäischen Bezügen, Berlin, 1984 e di N.G. Bartholdy, Friedrich Münter: videnskabsmand og frimurer, København, 1998. Per la famiglia rimando a U. Kjær, Frederik Münter og S. Petri kirke, Aarbøger for Nordisk Oldkyndighed og Historie, (2007) [ma 2010], 149-160.

5 Al riguardo G. Giarrizzo, Massoneria e illuminismo nell'Europa del settecento, Venezia, 1994, 224-274 e C. Francovich, Storia della massoneria in Italia. Dalle origini alla rivoluzione francese, Firenze, 1975, 309-354.

6 Per Münter numismatico: G. Galster, Frederik Münters Møntstudier, in Frederik Münter, et Mindeskrift, Hans Levned og Personlighed, I, 85-115; Id., Frederik Münter's Numismatic Studies, in Coins and History. Selected Numismatic Essays, Aarhus, 1959, 32-52 e, più recentemente, G. Gorini, Frederik Münter, Danish Numismatist: New Documents, in Scripta varia numismatico Tuukka Talvio sexagenario dedicata, Helsinki, 2008, 303-316. Ringrazio il Professor Gorini per avermi fatto avere copia di questo suo studio.

7 Disegnatore presso la zecca di Milano dal 19 novembre 1801 al 20 dicembre 1803 in questa data gli venne conferito l'incarico di occuparsi dell'appena fondato Gabinetto numismatico di cui, il 2 giugno 1808, divenne il conservatore; E.A. Arslan, Il Gabinetto numismatico dei civici Musei di Milano, in Compte rendu 22. Commission Internationale de Numismatique, 1975, 39. Per Cattaneo numismatico: F. Ghisalberti, Il numismatico Gaetano Cattaneo (1771-1841), Rendiconti dell'Istituto Lombardo di Scienze e Lettere. Classe di Lettere e Scienze Morali e Storiche 101, (1967), 761-782 e A. Savio e G. Della Ferrera, Il poliedrico Gaetano Cattaneo, fondatore del Gabinetto Numismatico di Brera, Archivio Storico Lombardo 7, s. 9, (1990), 347-374. 
Rossari. ${ }^{8}$ Dieci di queste lettere, inoltre, si sono conservate anche come minute della corrispondenza in uscita del Gabinetto Numismatico di Brera e si trovano oggi presso la Civica Biblioteca Archeologica e Numismatica di Milano. ${ }^{9}$ Come gli originali oggi in Danimarca neanche le minute milanesi hanno ricevuto sino ad oggi, forse proprio perché considerate esclusivamente "tecniche", quell'attenzione che invece meriterebbero. Nato come una corrispondenza ufficiale e d'ufficio, d'argomento prettamente numismatico, l'epistolario, come vedremo, toccò presto anche altri temi, ben più privati - non a caso, a conferma di un farsi sempre più personale della corrispondenza, si hanno quelle due lettere, non inviate dall'ufficio e assenti dal libro di copie di Milano e presenti solo a Copenaghen - e offre diversi spunti tanto per chi si occupi di Cattaneo quanto per chi studi Münter (e in particolar modo i rapporti di quest'ultimo con l'Italia).

8 Gli scritti di Münter si conservano presso Det Kongelige Bibliotek e, per quanto riguarda il materiale raccolto in quanto «agente massonico», presso l'archivio della Den Danske Store Landsloges, entrambi a Copenaghen. Pur avendone fatta richiesta non mi è stato permesso l'accesso a quest'ultimo fondo; i documenti che vi sono raccolti però, vi sono stati deposti il 7 aprile 1795 (N. Perrone, La Loggia della Philantropia. Un religioso danese a Napoli prima della rivoluzione. Con la corrispondenza massonica e altri documenti, Palermo 2006, 24) e non sembrano riguardare gli anni su cui si concentra la presente ricerca. Presso Det Kongelige Bibliotek si trovano invece diciotto volumi, alla segnatura NKS $16982^{\circ}$, contenenti: I-XVI, la corrispondenza in arrivo ordinata per ordine alfabetico degli scriventi; XVII, brutte e appunti; XIX, lettere sciolte di e a Münter. Franco Venturi (Illuministi italiani. Riformatori napoletani, Milano e Napoli, 1962, 780) ha parlato di un «archivio di Friedrich Münter», sempre a Copenaghen, ma Nico Perrone ( $\mathrm{La}$ Loggia della Philantropia, 238) ha fatto notare correttamente che questo archivio «non esiste». E' possibile però che Venturi si riferisse al fascicolo Privatarkiv Friederich Münter 1761-1830, Samlingspakke 1387, presso il già Rigsarkivet, oggi Statens Arkiver, di Copenaghen. Si tratta di un fascicolo minuto (una lettera di Paolo d'Ambrosio inviata da Napoli attorno al 1830, cinque lettere, 1822-1825 di Eduard Frederik von Saltza e alcuni appunti) collocato presso quella parte degli archivi di Stato danesi destinata a conservare «archivi personali». Durante le mie ricerche presso Det Kongelige Bibliotek di Copenaghen sono stato assistito dallo staff del centro manoscritti ed edizioni pregiate: Susanne Budde, Ivan Boserup, Erik Petersen e Anders Toftgaard. A tutti loro va il mio sincero ringraziamento per l'aiuto, la gentilezza e la disponibilità che mi hanno dimostrato al fine di facilitare le mie ricerche.

9 Le lettere sono regestate in La "Corrispondenza extra-ufficio" del Gabinetto Numismatico di Brera (1805-1851), e cura di R. La Guardia, Milano, 1985. Il personale della Civica Biblioteca Archeologica e Numismatica di Milano mi è stato di grande aiuto, in diverse circostanze, non solo facendomi dono di questo volume fuori commercio. Vorrei cogliere l'occasione per ringraziare in modo particolare Cristina D'Adda, Rachele Mauri ed Elisabetta Pernich. 


\section{2. «DANS LES RECHERCHES DE LA SCIENCE NUMISMATIQUE...», ASPETTI DI UN CARTEGGIO ERUDITO}

Tra il 1784 e il 1787 Frederik Münter soggiornò in Italia: ${ }^{10}$ il giovane studioso danese risiedette anzitutto a Roma ${ }^{11}$ e a Napoli, ${ }^{12}$ e per più breve tempo in Sicilia, ${ }^{13}$ ma, sulla via del ritorno in Danimarca, fece tappa anche in Lombardia, passando da Milano e da Pavia in particola-

10 Per il viaggio italiano di Münter, specialmente per quanto riguarda l'attività massonica: Francovich, Storia della massoneria in Italia, 381-433 e il diario già citato Frederik Münter, et Mindeskrift. Aus den Tagebüchern Friedrich Münters. Wander- und Lebrjabre eines Dänischen Gelebrten 1772-1787, II-IV. Sebbene sia oramai datato, merita comunque d'essere menzionato, e non solo per il suo valore quasi pionieristico, il saggio di B. Croce, Federico Münter e la massoneria di Napoli nel 1785-86, in Aneddoti di varia letteratura, 3 voll, Bari, 1954, III, 168-180.

${ }^{11}$ A Roma la sua attività si legò particolarmente a quella di Stefano Borgia, il quale si prodigò, a partire dagli anni Ottanta del Settecento per aiutare i giovani danesi che vi giungevano con lo scopo di poter esplorare le biblioteche vaticane e di perfezionare i propri studi. Al riguardo: Ø. Andreasen, Kardinal Borgia og de Danske $i$ Rom. A.C. Hviid, J.G. Chr. Adler, A. Birch, G. Zöega, Fr. Münter, N.I. Schow, T. Baden, Chr. Ramus, G. Wad, W. Fr. Engelbreth. 1779-1804, in Rom og Danmark gennem Tiderne, a cura di L. Bodé, 3 voll, København, 1935-1942, I, 268-314 e A. Hamilton: The Copts and the West, 1439-1822: the european discovery of the egyptian church, Oxford, 2006, 243-248. Una versione italiana dell'articolo di Andreasen, corredata da alcune note aggiuntive a cura di Karen Ascani, è stata pubblicata, con il titolo di Il Cardinale Borgia e i Danesi a Roma. A.C. Hviid, J.G. Chr. Adler, A. Birch, G. Zöega, Fr. Münter, N.I. Schow, T. Baden, Chr. Ramus, G. Wad, W. Fr. Engelbreth. 1779-1804, in Stefano Borgia e i Danesi a Roma, a cura di R. Langella, Velletri, 2000, 23-61.

12 Perrone, La Loggia della Philantropia.

13 E. Di Carlo, Dai Diari di Federico Münter (il suo soggiorno a Palermo), Archivio Storico per la Sicilia 4, (1938), 471-481; G. Snaiderbaur, La Sicilia nei diari di Friedrich Münter, Nuovi Quaderni del Meridione 81, (1983), 65-93 e 82 (1984), 91-107; H. Tuzet, Viaggiatori stranieri in Sicilia nel XVIII secolo, Palermo, 1988, 117-118 e V. Sciuti Russi, Riformismo settecentesco e Inquisizione siciliana. L'abolizione del terrible monstre negli scritti di Friedrich Münter, Rivista Storica Italiana 115, (2003), 112-148. Nel numero 28 (2001) degli Analecta Romana Instituti Danici sono stati pubblicati gli atti di un convegno dedicato a Frederik Münter e la Sicilia tenutosi il 26 novembre 1999, tra gli articoli forse più interessanti meritano d'essere citati almeno T. Fischer-Hansen, La conoscenza dell'Italia meridionale e della Sicilia greca in Danimarca nell'Ottocento: Frederik Münter e Saverio Landolina, 35-64 e P. Persiani, "Vedere biblioteche e vedere il mondo": Frederik Münter ricercatore di manoscritti ed $i$ suoi Fragmenta Patrum Graecorum, 83-100. Più di recente Tobias Fischer-Hansen ha offerto due ulteriori, preziosi, contributi alla questione con gli articoli Frederik Münter in Syracuse and Catania in 1786: Antiquarian Legislation and Connoisseurship in 18th Century Sicily, in Oggetti, uomini, idee. Percorsi multidisciplinari per la storia del collezionismo, a cura di G. Giarrizzo e S. Pafumi, Pisa, 2009, 117-137 
re. ${ }^{14}$ Che Cattaneo non abbia avuto modo di incontrare Münter durante i suoi anni italiani, e che non fosse stato neppure indirizzato a questi da qualcuno dei, molti, conoscenti che Münter aveva in Italia, appare evidente dalla prima lettera che il numismatico lombardo scrisse all'omologo danese nell'agosto del $1815 .{ }^{15} \mathrm{La}$ lettera (di fatto un consulto rivolto a Münter circa «la monnaie des Princes Latines d'Antioche»), in francese, e non in italiano (lingua che Münter padroneggiava e che impiegava per i rapporti con l'Italia), non contiene infatti alcun riferimento a amicizie comuni - che invece, come vedremo, ci sarebbero state - e si apre con una dichiarazione da parte di Cattaneo della conoscenza e del vivo apprezzamento per il lavoro scientifico di Münter: «c'est depuis bien long temps que je suivais une occasion de vous presenter mes hommages, \& de vous faire l'offre respectueux de mes services dans les recherches de la science numismatique que vous cultivez Monsieur avec tant de succès». ${ }^{16} \mathrm{La}$ corrispondenza tra $\mathrm{i}$ due, che come si è detto durerà dieci anni, rimarrà sempre legata ad aspetti eruditi, o meglio, ai problemi

e Frederik Münter og hans erfaringer fra det sicilianske antikvitetsvæsen $i$ slutningen af 1700-tallet, Aarbøger for Nordisk Oldkyndighed og Historie, (2007) [ma 2010], 91-106. Nello stesso volume è da vedersi anche il ricco articolo di M. Nielsen, Frederik Münter på udgravning $i$ Nola 1786, 107-148.

14 Escluse le pagine di Francovich poco o nulla si è scritto per quanto riguarda Münter nell'Italia settentrionale (Münter giunse da Vienna, nel 1784, passando quindi dal Triveneto mentre tre anni dopo, al momento di lasciare il paese, visitò Lombardia e Piemonte sulla via della Savoia); significative eccezioni sono gli studi di R. Gaeta, Per la storia della massoneria nel'700 veneto. Il soggiorno padovano di Friedrich Münter illuminato bavarese, Critica storica 23, (1981), 180-198; di A. Stella Friedrich Münter e il suo soggiorno veneto (dicembre 1784). Considerazioni su alcune ipotesi da lui formulate sull'idioma "cimbro", in 700 anni di storia cimbra veronese, a cura di G. Volpato, Verona, 1987, 165-168, e di S. Ferrari, Libri, storia e Altertumswissenschaft. Amadeo Svaier e gli eruditi danesi a Venezia sul finire del Settecento, Analecta Romana Instituti Danici 28, (2001), 135-152.

15 Gaetano Cattaneo a Frederik Münter, Milano 17 agosto 1815, Det Kongelige Bibliotek, NKS $16982^{\circ}$, IV, 5, lettera 517.

16 Anche Cattaneo era comunque già noto a Münter in quanto numismatico per lo meno dal 1812, come dimostra la sua citazione in una lettera inviatagli da Georg Friedrich Creuzer. Georg Friedrich Creuzer a Frederik Münter, Heidelberg 20 settembre 1812, in Frederik Münter, et Mindeskrift. Briefwechsel Friedrich Münters; Europäische Beziebungen eines dänischen Gelebrten 1780-1830, V, 181. Anche per quanto riguarda la conoscenza di Münter da parte di Cattaneo, come si evince dalle minute milanesi, era stato il mondo tedesco a fare da tramite, specialmente in seguito all'acquisto da parte del gabinetto milanese, nel 1811 a Dresda, della collezione di monete cufiche appartenuta a Johann Gottfried Lipsius. 
tipici della comunicazione tra dotti tra Sette e Ottocento, quali il reperimento di libri, lo scambio di informazioni, l'aggiornamento sul lavoro e sulla vita dei colleghi stranieri così come sulle iniziative culturali ed editoriali dei rispettivi paesi. ${ }^{17}$ Le ultime righe della lettera del 18 giugno $1816^{18}$ sono un ottimo esempio della complessità, forse ancora più che della vastità,${ }^{19}$ dei problemi scientifici affrontati nel carteggio, e delle difficoltà che, per colpa della distanza e della lentezza nelle comunicazioni, caratterizzavano questo genere di scambi:

ed a proposito di un tale Museo [il 'Regio Museo' di Copenaghen] ${ }^{20}$ sarei pure curioso di sapere a quali mani sia affidato, e se gli studj numismatici

17 Münter informerà, non a caso, i suoi conoscenti delle notizie fattegli avere da Cattaneo circa i suoi studi e, più in generale, circa i risultati scientifici dei dotti milanesi. Se ne hanno esempi nella lettera a Joseph Hammer, [Copenaghen] 30 dicembre 1817, «Cattaneo in Mailand wird aber Auskunft schaffen können, und ich halte es für wichtig dieß zu wissen, um das Zeitalter etwas genauer bestimmen zu können, in dem eine solche Sitte eingefürt war», ibidem, 356; o in quella a Jeremias David Reuß, Copenaghen 31 luglio 1819, «ohne Zweifel haben Sie schon die in Mailand erschienene Ausgabe des Chronici Eusebiani. Der in dieser übersetzte Armenische Text soll viel reichhaltiger seyn, als der griechische. Mein Exemplar ist wahrscheinlich jezt in Frankfurt, wohin Cattaneo in Mailand es mit Kaufmannswaaren geschickt hat», ivi, VI, 129; o, ancora, in quella a Friedrich von Schlichtegroll, Copenaghen 27 febbraio 1818, «meine Samlung erhält selten Zuwachs: ich bin zu weit von den Quellen entfernt. Doch habe ich jetzt über 3000 griechische Münzen. Neulich schickte mir Cattaneo einige dreissig aus den Mailändischen Doubletten gegen norddeutsche, nordische und englische aus dem Mittealter.», ivi, VI, 206.

18 Gaetano Cattaneo a Frederik Münter, Milano 18 giugno 1816, Det Kongelige Bibliotek, NKS $16982^{\circ}$, IV, 5, lettera 519.

19 Da parte di Cattaneo gli interessi furono esclusivamente numismatici mentre da parte di Münter si riscontrano orizzonti più vasti, come anche lo stesso Cattaneo riconoscerà parlando di le «di Lei ricerche Archeologiche» (Milano, 18 giugno 1816, ivi, lettera 519) e dei «vostri studj archeologici» (Milano, 24 marzo 1819, ivi, lettera 526). Al di là degli aspetti numismatici, e impiegando il tornasole, certo non sempre esente da limiti, delle missive di risposta, possiamo dedurre che Münter chiese aiuto a Cattaneo anzitutto, ma non solo, per trattare temi di storia della Sardegna (da collocarsi all'interno di più vaste ricerche attorno alla religione di Cartagine), per studiare gli ordini cavallereschi e in particolar modo il danese Ordine dell'Elefante, quindi per la storia dei Templari, per quella del testo biblico e, ancora, per lo studio dei codici greci già intrapreso negli anni italiani, chiedendo pure, ma invano, assistenza per ricerche da effettuarsi presso la Biblioteca Ambrosiana (Milano, 21 giugno 1817, ivi, lettera 522). La lettera 526 non reca né data né luogo, a differenza delle minute milanesi che ci permettono di collocare con sicurezza il testo. Milano, Civica Biblioteca Archeologica e Numismatica, Corrispondenza extra-ufficio, 183.

$20 \mathrm{Si}$ tratta con tutta probabilità della nascita, nel 1807, della Kongelige 
abbiano cossì molti cultori. Mi dica pure di grazia se sia vera l'infausta notizia qui sparsa da un tale che fece per Maggio passaggio da Milano, della morte del Professor Tychsen di Rostock $?^{21}$ Se ha occasione, la prego di volermi procurare gli Atti dell'Accademia delle Scienze di Copenaghen, e tutte l'opere Cufiche di Hallenberg. ${ }^{22}$ Mi dimenticava di dirle che non ho alcuna medaglia fra' duplicati del Basso Impero coll'iscrizione di RAV o RAVEN. Non mi sarà però difficile col tempo di procurargliene. ${ }^{23}$

Fu sempre un aspetto di carattere erudito che, con tutta probabilità, provocò, a distanza di dieci anni, la fine del carteggio e dell'amicizia tra Münter e Cattaneo. L'ultima lettera in nostro possesso, inviata il 18 giugno 1825 da Milano, indicativamente in francese, si apre, in media res e in aperto contrasto con gli incipit ben più cerimoniosi e amichevoli delle lettere precedenti, ${ }^{24}$ con l'affermazione stupita «j'ai été un

Kommission til Oldsagers Opbevaring (Commissione reale per le Antichità) dalle cui collezioni fu poi istituito il Nationalmuseet di Copenaghen. Al riguardo si veda il numero monografico degli Acta Archaeologica 78, (2007), Birth of a World Museum. Specialmente per il ruolo di Münter in questa istituzione si veda l'articolo di T. FischerHansen, Frederik Münter and the Kongelige Oldsagscommission of 1807 alle pagine 179-181. Ricchi di spunti sono poi gli interventi di T. Benedikte Jakobsen, Arbejdet $i$ Oldsagskommissionens første år, Aarbøger for Nordisk Oldkyndighed og Historie (2007) [ma 2010], 161-192 e, nello stesso numero, N.G. Bartholdy, Oldsagskommissionen og frimureriet - en kulturel symbiose, 193-200.

21 Oluf Gerhard Tychsen, orientalista tedesco, fu il vero e proprio fondatore della numismatica araba e mediorientale in Europa. Al riguardo: H. Tröger, Die Erwerbung des Nachlasses von Oluf Gerhard Tychsen, Orientalist und Bibliothekar durch die Universitätsbibliothek Rostock, Rostock, 1990.

${ }^{22} \mathrm{Si}$ tratta di Jonas Hellenberg, storico e antiquario svedese che curò una edizione delle collezioni di monete cufiche possedute dal gabinetto numismatico dell'Università di Upsala: Collectio nummorum cuficorum, quos aere expressos, addita eorum interpretatione, subjunctoque alphabeto cufico, a cura di I. Hallenberg, Stockholm, 1800. Aveva poi edito il volume Ex Occasione Nummi Cufici de Nominis Dei Gud in Suio-Gothica Cognatisque Linguis Origine, Stockholm, 1796 mentre nel 1822 pubblicherà l'importante studio in due tomi, Numismata orientalia, Upsala.

23 Gaetano Cattaneo a Frederik Münter, Milano, 18 giugno 1816, Det Kongelige Bibliotek, NKS $16982^{\circ}$, IV, 5, lettera 519.

${ }^{24}$ Proprio il tema dell'amicizia, che ritorna anche nella lettera al Rossari da cui si è partiti, aveva presto caratterizzato il rapporto epistolare. Cattaneo, il 18 giugno 1816 (ibidem), aveva scritto a Münter «mi ascriva al numero de' suoi ammiratori, e se me ne credo degno anche in quello degli amici». Un calore che doveva però essere stato ricambiato da Münter visto che il 30 giugno 1817 (ivi, lettera 523) Cattaneo si schernì affermando «vi chieggo perdono pel tuono confidenziale che mi vedete assumere nella presente mia lettera. Ciò non è che il frutto delle cordiali espressioni onde sono pieni $\mathrm{i}$ 
peu surpris à la lecture d'un paragraphe d'une lettre de votre main». La prima facciata continua poi, di fatto, con un lungo elenco di scuse, o, a seconda dei punti di vista, di ragioni, per quello che Cattaneo stesso chiama «mon long silence». La mancanza della lettera di Münter non permette di affermare con assoluta certezza cosa questi avesse rimproverato a Cattaneo ma a giudicare dal contenuto della risposta in nostro possesso, ciò dovette riguardare l'aiuto - richiesto, promesso e poi invece mancato da Cattaneo - per assistere Münter nelle ricerche di cui aveva grande bisogno presso gli archivi di Mantova. Le ricerche riguardavano la storia del Elefantordenen (l'Ordine dell'Elefante), il più prestigioso ordine cavalleresco della Danimarca, che in quel frangente interessava in modo particolare l'ecclesiastico nordico il quale, solo pochi anni dopo, avrebbe pubblicato i propri studi sugli ordini cavallereschi danesi nel volume Undersøgelser om de danske Ridderordeners Oprindelse (1822). Nel testo andato in stampa solo due magre pagine ${ }^{25}$ sono dedicate al fatto che Ludovico III Gonzaga fosse stato insignito da Cristiano I di Danimarca dell'Elefantordenen durante il viaggio in Italia di questi nel $1474 ;{ }^{26}$ al riguardo Münter era stato costretto ad affermare che, forse, solo uno studio dell'archivio di Mantova avrebbe potuto dare in futuro qualche frutto per meglio comprendere sia la visita di

fogli che mi faceste l'onore di diriggermi». Sicuramente le gentilezze di Münter dovettero contribuire a far assumere in Cattaneo quel tono amichevole di cui si è detto; d'altro canto va forse fatto notare come le lodi di Münter riferite da Isimbardi, Saurau e Tamburini dovettero per lo meno suggerire a Cattaneo l'importanza e la fama del corrispondente e forse, anche involontariamente, lusingarlo all'idea di potersene dire, lui pure, amico.

${ }^{25}$ F. Münter, Undersøgelser om de danske Ridderordeners Oprindelse, København, 1822, 74-76.

${ }^{26}$ Le casate dei Gonzaga e degli Oldenburg, la casa regnante danese, erano unite da legami matrimoniali e dinastici avendo sposato, Ludovico III Gonzaga, Barbara di Brandeburgo, sorella di Dorothea, regina di Danimarca. Nel 1474 Cristiano I scese in Italia in pellegrinaggio e, sulla via di Roma, passò da Mantova dove venne accolto con tutti gli onori. In generale per i rapporti tra le due case regnanti: B. Holm, L'incontro di due mondi: Cristiano I di Danimarca e la casata dei Gonzaga, in I Gonzaga e l'Impero. Itinerari dello spettacolo, a cura di U. Artioli e C. Grazioli, Firenze, 2005, 4152 e J. Lindbæk, Dorothea, Kristiern den forsters dronning, og familien Gonzaga, Historisk Tidsskrift 3, n.s., (1900-1902), 455-512. Per il viaggio in Italia di Cristiano I: V. Ettig, R. Signorini e B. Wederlin, Fra Christian I's Italiensrejse 1474, København, 1984 e i due articoli di R. Signorini, Federico III e Cristiano I nella camera degli sposi del Mantegna, Mitteilungen des Kunsthistorischen Institutes in Florenz 18, (1974), 227-246 e Id., Cristiano I in Italia, Il Veltro 25, (1981), 23-58. 
Cristiano che le circostanze del conferimento della decorazione a Ludovico. ${ }^{27}$ Ritengo verosimile che Münter, nella lettera che non possediamo, abbia rinfacciato a Cattaneo, più o meno velatamente, questa mancanza. Nella missiva del 18 giugno 1825 Cattaneo affermava infatti, «à ma justification [...] je m'étais flatté de pouvoir vous procurer, d'áprés les Archives de Mantoue, que la personne qui s'en était chargé, m’a toujours amusé de paroles, pendant longtemps, et à la fin n'a rien su me dire». Münter aveva però sollecitato l'aiuto di Cattaneo già diverso tempo prima. La lettera del 25 gennaio 1823 si apre infatti con una richiesta di venia per il fatto che «tempo fa mi scriveste per dirmi che desideravate di far praticare qualche ricerca nell'Archivio della Casa Gonzaga di Mantova». La precedente missiva di Cattaneo è datata aprile 1819; Münter aveva insomma scritto molto prima al numismatico milanese, e sebbene non possiamo essere sicuri sulla data parrebbe più che probabile, se non quasi certo, che lo abbia fatto quando il suo libro era ancora lungi dall'essere chiuso; il silenzio di Cattaneo lo aveva però costretto a lasciare di fatto incompiuta la parte riguardante Cristiano I e i Gonzaga. L'irritazione che con tutta verosimiglianza Münter manifestò risulta poi ancora più comprensibile tenendo presente le continue offerte fatte da parte di Cattaneo, «in contraccambio» della generosità del danese, «a servirla in tutto quello in cui io possa trovarmi capace». ${ }^{28}$ Non sappiamo se Münter replicò mai alla lettera di Cattaneo del giugno 1825, parrebbe di no, sicuramente Cattaneo smise di scrivere, esprimendo un silenzio risentito che non gli permise neppure di comunicare a Münter la morte dell'«incomparabile Tamburini» avvenuta a Pavia il 14 marzo 1827. I sentimenti di Münter si dovettero andare rasserenando però se il 19 aprile 1828, corrispondendo con Ignaz Heinrich von Wessenberg, ${ }^{29}$ il quale era in procinto di dirigersi in Italia e pensava di transitare da Milano, gli rivolse la richiesta, anzi letteralmente la preghiera, di salutarvi alcuni suoi conoscenti quali il conte Carlo Innocenzo Isimbardi, Luigi Tosi, Carlo Ottavio Castiglioni, ma anche

27 «Archivet i Mantua, hvis det endnu er til, maatte dog vel kunne give nærmere Oplysning om Kongens Ophold der, maaskee og om hans Foræring til Markgreven», Münter, Undersøgelser om de danske Ridderordeners Oprindelse, 76.

28 Gaetano Cattaneo a Frederik Münter, Milano 18 gennaio 1817, Det Kongelige Bibliotek, NKS $16982^{\circ}$, IV, 5, lettera 520.

${ }^{29}$ Frederik Münter a Ignaz Heinrich von Wessenberg, Copenaghen 19 aprile 1828, Frederik Münter, et Mindeskrift. Briefwechsel Friedrich Münters, VI, 369. 
Cattaneo: «Sie auch nach Mailand, so bitte ich Sie, die Grafen Isinbardi u. Castiglione, ${ }^{30}$ und Cattaneo von mir bestens zu grüssen; ebenfalls Monsignore Tosi, Bischof von Pavia, falls Sie ihn sehen. Ich machte seine Bekanntschaft im lombardischen Seminario bei Zola und Tamburini». La richiesta rivolta a von Wessenberg ci dice però anche qualcos'altro circa Münter e gli amici milanesi; Isimbardi, che tra i milanesi era stato tra i più vicini a Münter, era morto infatti già nel 1824 . Con la fine del rapporto epistolare con Cattaneo quindi, Münter doveva aver perso ogni contatto con Milano. Al di là degli aspetti numismatici, la corrispondenza tra Cattaneo e Münter assume grande rilevanza proprio per poter provare a gettare nuova luce sui legami tra Münter e gli ambienti lombardi, oltre che per meglio comprendere le dinamiche del suo passaggio in Lombardia, passaggio di cui, escluse le pagine del diario, molto poco è noto, specialmente se lo si paragona alle ben più documentate permanenze a Napoli e in Sicilia.

\section{GAETANO CATTANEO COME CORRISPONDENTE E INTERMEDIARIO}

Il 18 aprile 1816 Cattaneo inviò una seconda lettera a Münter. Qui, passando all'italiano, su presumibile invito di quest'ultimo, Cattaneo diede ragione del ritardo nel rispondere affermando che «il desiderio che mi nacque di darle qualche circonstanziata notizia degli amici de' quali Ella conserva così viva rimembranza, mi fece nascere il pensiero di chiederle ad essi medesimi indicando lo scopo cui era diretta la mia ricerca». ${ }^{31}$ Münter, nella propria risposta, doveva quindi aver chiesto lumi circa gli amici lombardi, anzitutto pavesi e milanesi, incontrati oramai vent'anni prima, tra il gennaio e il febbraio del 1787. Cattaneo si mostrò felice di informare Münter al meglio delle sue capacità e, un po' per le sempre nuove richieste di Münter, un po' sua sponte, presto si ritrovò a fare da vero e proprio tramite tra i conoscenti che

30 Si tratta di Carlo Ottavio Castiglioni, orientalista e numismatico milanese, collaboratore di Cattaneo all'edizione della collezione di monete cufiche di Brera, di cui curò un primo catalogo: Monete cufiche del museo di Milano, Milano 1819. Si veda: A. Paredi, Carlo Ottavio Castiglioni, in Dizionario Biografico degli Italiani, 71 voll, Roma, 1960-, XXII, 137-138.

31 Gaetano Cattaneo a Frederik Münter, Milano 18 aprile 1816, Det Kongelige Bibliotek, NKS $16982^{\circ}$, IV, 5 , lettera 518. 
il dotto danese aveva in Lombardia, al punto che, come vedremo, tale "aggiornamento" divenne in poco tempo, insieme alla numismatica e alla discussione erudita, il vero tema della corrispondenza tra i due.

Tra le persone di cui Münter chiese conto, alcune dovevano essere poco note a Cattaneo che, dopo averle interpellate o dopo essersi informato sulla loro condizione, diede al corrispondente danese solo dei brevi sunti; è il caso per esempio di Luigi Bossi, ${ }^{32}$ e di Giacomo Rezia. ${ }^{33}$ Del primo Cattaneo comunicò a Münter che

Monsignor Bossi, non è più né Monsignore né prete. All'epoca della nostra politica pazzia egli abbandonò lo Stato ecclesiastico e gettassi nel vortice degli affari, a tal che fu del Corpo Legislativo, Prefetto delle Biblioteche degli Archivi, ed infine Agente diplomatico. Ora egli non è più nulla di tutto ciò, e vive ritiratissimo, e ruinato. ${ }^{34}$

\section{Mentre del secondo informò il suo interlocutore che}

anch'egli abbandonò la Sua Carica ond'era insignito nell'Università di Pavia per correre lo Stadio rivoluzionario. Si ridusse però, dopo il suo primo vagare di pensieri, a prendere servizio nelle truppe della Repubblica in qualità di Medico Militare, ed in breve salì al grado di Medico in Capo. Ora anch'egli si è ritirato, e vive a Menaggio sua patria sul Lago di Como.

Ben altro spazio venne invece dato nella lettera dell'aprile 1816 a quelle personalità con cui Cattaneo aveva un qualche legame, come Pietro Tamburini, di cui diremo fra poco, e il conte Isimbardi, patrizio milanese e collezionista numismatico che Cattaneo ben conosceva:

si è data una fortunatissima occasione - scrive infatti Cattaneo a Münter che il Marchese Isimbardi ch'Ella m'impose di riverire sia appunto il Direttore Generale dell'Amministrazione delle Monete e come tale mio Superiore; essendo l'I.R. Gabinetto di Medaglie unito all'Ufficio da lui dipendente. Egli fu penetratissimo dalla di lei grata memoria, e mi disse tante

32 L. Sebastiani, Luigi Bossi, in Dizionario Biografico degli Italiani, XIII, 323-327.

33 L. Bonandrini, Giacomo Rezia, in "...parlano un suon, che attenta Europa ascolta”. Poeti, scienziati, cittadini nell'Ateneo pavese tra Riforme e Rivoluzione, Pavia, 2000, 247-254.

34 Gaetano Cattaneo a Frederik Münter, Milano 18 Aprile 1816, Det Kongelige Bibliotek, NKS $16982^{\circ}$, IV, 5, lettera 518.

35 Ibidem. 
belle e preziose cose sul di lei conto, ch'io non potei che raddoppiare il sentimento di stima la di lei fama aveammi già fatta nascere. M'incaricò di farle i più cordiali saluti, e di offrirle la Sua servitù. La di lui Madre è infatti morta da varj anni, come Ella aveva avuto presentimento.

Isimbardi tornerà nel carteggio in diverse altre occasioni; saluterà Münter attraverso Cattaneo più volte, ${ }^{36}$ accompagnato, a partire dal 1 maggio $1817,{ }^{37}$ da Franz Joseph Conte di Saurau, all'epoca governatore della Lombardia e che aveva conosciuto Münter a Vienna ${ }^{38}$ oltre vent'anni prima:

ho fatto i di Lei saluti al Marchese Isimbardi, che li contraccambia con usura. Non ho pure tralasciato di fare anche questi al Signor Conte di Saurau, ed ho avuto luogo di accorgermi in quanta stima ella sia tenuta presso quel degno Personaggio. Egli incaricami di manifestarle l'infinito piacere che ha provato nello scorgere tuttavia impressa la sua memoria nel di lei cuore. Egli non lo ha avuto meno presente, ed ha sempre sentito la più viva gioia tutte le frequenti volte che ha sentito onorare il di Lei nome, nelle varie parti dell'Europa da lui percorsa, da che' non ebbe più occasione di vederla. ${ }^{39}$

Anche se non citati necessariamente per nome i conoscenti italiani torneranno ancora nel carteggio come quando, nel gennaio del 1817, Cattaneo inviò al dotto danese $\mathrm{i}$ «saluti cordialissimi degli amici che tiene in questo paese»...$^{40}$ Ben più spazio, come si accennava, lo ebbe però Pietro Tamburini.

Nominato spessissimo, il prelato bresciano può essere considerato un vero e proprio partecipante al carteggio visto che due suoi lunghi passaggi vennero trascritti verbatim da Cattaneo e da questi inviati a Münter; sappiamo inoltre di una lettera, oggi perduta, che il prelato danese fece recapitare a Tamburini, sempre attraverso Cattaneo. Il

36 Gaetano Cattaneo a Frederik Münter, Milano 21 giugno 1817 e 29 agosto 1817, Det Kongelige Bibliotek, NKS 1698 2, IV, 5, lettere 522 e 524.

37 Gaetano Cattaneo a Frederik Münter, Milano 1 maggio 1817, ivi, lettera 521. I saluti saranno ripetuti anche il 21 giugno 1817 e il 29 agosto 1817, ivi, lettere 522 e 524 .

$38 \mathrm{Si}$ erano incontrati diverse volte nell'ottobre del 1782 a Vienna, Frederik. Münter, et Mindeskrift, Aus den Tagebüchern Friedrich Münters, II, 99, 101 e 118-119.

39 Gaetano Cattaneo a Frederik Münter, Milano 1 marzo 1817, Det Kongelige Bibliotek, NKS $16982^{\circ}$, IV, 5, lettera 521.

40 Gaetano Cattaneo a Frederik Münter, Milano 18 gennaio 1817, ivi, lettera 520. 
primo degli stralci in questione si trova già nella missiva del 18 aprile 1816 e merita d'essere riportato in extenso:

Di Tamburini io non saprei darle migliore contezza che trascrivendole letteralmente ciò che di se medesimo mi scrisse tosto ch'io ne lo richiesi in di Lei nome. Eccolo: "Circa le notizie che riguardano la mia persona massimamente nelle vicende dei decorsi tre lustri, manderò forse a suo tempo al rispettabile amico, il dottissimo Signor Münter, alcune lettere latine, nelle quali le ho descritte in dettagli. Per ora dirò che nella rivoluzione delle cose politiche e degli umani cervelli Iddio mi volle sul campo di battaglia per sostenere colle mie deboli forze i principj che si sovvertivano, della buona morale, e del diritto naturale, e sociale. Spinto dall'alto mare teologico verso terra fui obbligato a radere il lido. Sostenni per più anni l'impeto della licenza filosofica su questa Università, ed il Signore mi diede forza e coraggio di sostenerla con felice riuscita. Ho stampati in questo frattempo sette volumetti di Filosofia morale, e di Diritto Universale, e questi in lingua italiana, poiché per desiderio dei tempi era esule dall'Università la lingua latina. In quest'anno però ho pubblicato gli Elementi del Naturale diritto in latino. Pubblicai parimenti due volumetti di lettere Teologico-politiche in occasione della Rivoluzione francese, e del libro di Spedalieri uscito in Roma Sui diritti dell'uomo ${ }^{41}$ e finalmente scrissi due letterine sulla vana pretesta di alcuni filosofi, di separare la Religione dal Sistema politico, e sulla necessità della sorveglianza politica sul pubblico insegnamento ecclesiastico. In tal modo io corsi questi anni di guerra, e di torbidi, facendo tacer l'amor del ritiro a fronte del dovere di cittadino e di sacerdote a vantaggio della Religione e dello Stato. Ora tocco l'anno ottuagesimo dell'età mia, e continuo le mie lezioni su quest'Università, e per facilitare il disimpegno della mia Catedra, attesa l'età mia avanzata, ho provveduto un legnetto con un eccellente cavallo, che ne' giorni delle lezioni mi conduce all'Università, e di là mi riconduce a casa. La mia salute è discreta a fronte del peso degli anni, che mi gravita sulle spalle, e l'abitudine contratta d'insegnare per 54 anni tra Brescia, Roma e Pavia mi

${ }^{41}$ E' questa la ben nota, e molto indagata, polemica tra Tamburini e Spedalieri: al riguardo i due studi di G. Giraldi, La sovranità popolare nella polemica SpedalieriTamburini, Rivista internazionale di filosofia del diritto 32, (1955), 297-317 e Il vero volto di Nicola Spedalieri e Pietro Tamburini, Rivista internazionale di filosofia del diritto 34, (1957), 597-622; E. Traniello e E. Passerin d'Entrèves, Ricerche sul tardo giansenismo italiano, Rivista di storia e letteratura religiosa 3, (1967), 280-313; G. Ruggieri, Teologia e società. Momento di un confronto sul finire del '700 in riferimento all'opera di Nicola Spedalieri, Cristianesimo nella storia 2, (1981), 437-486; E. Pii, Un aspetto della reazione cattolica: il caso Spedalieri, Cristianesimo nella storia 10, (1989), 251-272 e, più recentemente, F. Traniello, Tamburini e Spedalieri: i dilemmi della sovranità, in Pietro Tamburini e il giansenismo italiano, 85-105. 
fa superar di buon grado la fatica. Ora si attende il ristabilimento della Facoltà Teologica esule da gran tempo da quest'Università; e con essa si aspetta un compiuto piano degli Studj, in tutti i rami delle Scienze umane e divine. Confido nei lumi e nello zelo del nostro Governo di vedere al trammontar de' miei giorni perfezionata la organizzazione di questa celebre Università con un piano di Studj analogo alla indole delle Scienze, ed ai bisogni delle nostre Provincie. Ecco le poche notizie ch'io posso dare all'ottimo amico". Non dubito punto ch'Ella non mi sappia buon grado di averle trascritto per esteso questo nitido ed ingenuo compendio della vita passata in quest'ultimi 20. anni da un uomo cotanto rispettabile, e celebre in Europa. ${ }^{42}$

La citazione di Tamburini fu solo la prima attestazione di una presenza che andrà facendosi quasi costante nella corrispondenza tra Cattaneo e Münter. Il 13 maggio 1819, rispondendo evidentemente a un sollecito al riguardo da parte di Münter, Cattaneo lo rassicurò: «il buon Tamburini è sempre vegeto, stimato ed amato, ad onta dei fulmini del Vaticano. Quando Sua Maestà visitò l'Università di Pavia, nella quale Tamburini è Direttore di una delle tre Facoltà, gli domandò come andavano i suoi affari colla Corte di Roma. Il buon prete rispose: "Maestà ora sono troppo vecchio, quindi ho levato l'assedio" ${ }^{43}$ L'affermazione fatta al Rossari da cui siamo partiti, e cioè che Münter gli chiedesse di salutare per lui Tamburini «ogni volta che ha l'amicizia di scrivermi», che si sarebbe tentati di credere un'iperbole, pare insomma rispecchiare il vero. Fu tra il 2 marzo, data della lettera al Rossari in cui non se ne fa cenno, e il 24 dello stesso mese, quando cioè Cattaneo tornerà a rivolgersi a Münter, che il numismatico milanese dovette ricevere una nuova missiva dal suo omologo danese in cui era accluso anche un biglietto oggi perduto per Tamburini. Il contenuto della nota si può comunque dedurre dalla successiva lettera di Cattaneo in cui venne trascritta, ancora una volta, la risposta dell'ecclesiastico bresciano. Il testo merita, anche in questo caso, di essere riportato in tutta la sua prima parte:

42 Gaetano Cattaneo a Frederik Münter, Milano 18 aprile 1816, Det Kongelige Bibliotek, NKS $16982^{\circ}$, IV, 5, lettera 518. Questa lettera è l'unica ad essere già stata edita e si trova in Frederik Münter, et Mindeskrift. Briefwechsel Friedrich Münters, V, $170-171$.

43 Gaetano Cattaneo a Frederik Münter, Milano 13 gennaio 1819, Det Kongelige Bibliotek, NKS $16982^{\circ}$, IV, 5 , lettera 525. 
Amico Veneratissimo,

Il preziosissimo foglio che vi degnaste scrivermi in data dell' 8 Febbraio p.p. mi ha riempito di contentezza, dimostrandomi nel modo il più lusinghevole quanto favorevolmente accogliate ciò che da me vi perviene, e quale conto facciate di quel poco ch'io sono in grado di fare a giovamento dei vostri studj archeologici. Era perciò mia spontanea intenzione di manifestarvene immediatamente la mia intima riconoscenza; ma non volli farlo prima di aver comunicato lo stesso vostro foglio al buon Tamburini. Gli acclusi infatti il medesimo in una mia, persuaso di cagionargli una gratissima sensazione. Quale sia stato l'effetto di essa, vi sarà facile di rilevarlo dal contesto della sua risposta ch'io mi pregio di trascrivervi: "Io non posso esprimerle abbastanza quanto mi sia stato caro il grazioso paragrafo ch'Ella mi comunica, dell'eruditissimo Monsignor Vescovo, sì per la memoria che l'illustre soggetto serba di me, come per le notizie ch'egli mi dà dei felici progressi, che mediante la cura dei Principi promettono nell'Alemagna Cattolica i buoni principj del diritto Ecclesiastico. Faccia il Signore che questi germi coltivati a dovere portino il frutto desiderato in tutta la Chiesa Cattolica, come fa sperare il movimento che prendono nell'Alemagna le cose ecclesiastiche. Un aspetto contrario hanno fra noi diffatti studj, e n'è una prova la guerra arrabbiata che si muove in Milano all'editore delle Opere mie per impedire la continuazione a fronte del grazioso decreto dell'Augusto Nostro Sovrano, che ne permette l'intera riproduzione. Oserei pregarla di spedire all'esimio Prelato una o due copie dell'invito, che accludo, dell'Editore, per l'associazione alla edizione intrapresa delle opere mie. Fra le varie operette che in essa si annunziano ve ne potrebbe essere alcuna, che più incontrasse il di lui genio, ed allora impegnerei la di lei compiacenza per fargliela avere. Sinora sono uscite in Milano nella ristampa due operette, l'una col titolo Vera idea della Santa Sede, e l'altra: Analisi delle prescrizioni di Tertulliano. Ciò è bastato per eccitare i più alti clamori come in città stretta d'assedio, che sia non già levato, ma via forzato. Io frattanto prego Lei vivamente di recare a quel dottissimo Vescovo i miei più vivi ringraziamenti per l'affetto, ch'egli si degna di conservarmi, e per l'interessamento ch'Egli si prende delle cose mie. Sono poi sensibilissimo alla di lei umanità che si compiace di tenermi vivo nella memoria di lui, favore per me singolare e per cui le professo \&." Appena ricevuta questa risposta, calcolando che voi non avreste certamente bilanciato a sacrificare sette franchi per procacciarvi il piacere di ricevere sollecitamente le due indicate opere, mi sono determinato ad acquistarle, e a unirle, ${ }^{44}$

44 Sebbene non vi sia traccia della seconda ho avuto modo di rinvenire, presso Det Kongelige Bibliotek di Copenaghen, una edizione della Vera idea della Santa Sede, Milano 1818, di Tamburini recante una annotazione autografa di Münter, che recita: «Friderici Münteri munu Cajetani Cattanei Mediolanensis 1819» e che registra, correttamente, il volume come dono di Cattaneo, pur se su invio di Tamburini. La biblioteca di Münter venne venduta all'asta dopo la sua morte, il 31 marzo 1831, rendendo così le ricerca sui volumi da lui posseduti particolarmente difficile. Sopravvive in compenso il 
insieme ai due Manifesti inviatimi da Tamburini, all'Eusebio di Mai, ${ }^{45}$ che in que-

catalogo preparato per l'asta: Bibliotheca Münteriana, sive: Catalogus librorum quos reliquit Fredericus Münter, København, 1830. Qui come in seguito, quando si citerà da questo testo, si è preferito riportare le descrizioni così come vi sono date, abbreviazioni e corsivi compresi, aggiungendo di seguito la pagina del catalogo e il numero con cui sono state registrate. Vi compaiono diversi libri di Cattaneo: «(G. Cattaneo) Osserv. sopra un Frammento antico di Bronzo di Greco Lavoro rappresentante Venere. Milano 1819», ivi, 520, n. 54; «G. Cattaneo, Equeiade, monumento antico di Bronzo del museo Ungherese, Milano 1819», ivi, 538, n. 413; «Descr. di alc. Monete Cufiche del Museo di S. Mainoni. Milano 1820», ivi, 549, n. 688; «G. Cattaneo Catalogus numorum pop. urb. \& regum in Museo reg. offcinæ monetariæ Mediolanensis, Mediol 1813», ivi, 580, n. 1438; «Obss sulla Descr. di alc. monete cufiche, Mil. 1821», ivi, 582, n.1464 e «Lettera di G.C. a Sestini sopra II. Medaglie Greche del R. Gabinetto di Milano, Mil. 1811», ibidem, n. 1472. E’ poi presente il testo «(G. Cattaneo) Monete Cufiche del J.R. Museo di Milano, Mil 1819», ivi, 549, n. 687. Il volume in questione non è però di Cattaneo quanto quello di Carlo Ottavio Castiglioni di cui si è parlato alla nota 30. La ragione della confusione credo sia da ascriversi al fatto che il testo, che non reca nel frontespizio il nome di Castiglioni, sia preceduto da una introduzione di Cattaneo. Potrebbe anche darsi però che un'annotazione a margine di Münter circa il fatto che gli fosse stato donato da Cattaneo passa aver indotto in errore il compilatore del catalogo d'asta.

45 Si tratta della traduzione latina del Chronicon di Eusebio di Cesarea, sopravvissuta in armeno e scoperta da Angelo Mai, citata nella lettera di Münter a Reuß che si è riportata alla nota 17 . Il volume s'è conservato nella biblioteca di Münter, «Eusebii Pamphili E Samuelis Aniensis Chronica Ang. Majus et Joh. Zohrabus edid. notisque illustr. Mediol. 1818», Bibliotheca Münteriana, 70, n. 806. L'opera era stata molto apprezzata da Münter che l'aveva fatto sapere a Cattaneo il quale aveva subito informato Mai in una lettera inviatagli da Milano il 27 maggio 1820: «Mi affretto a pregare il Signor Conte Alborghetti del favore di trasmetterle l'accluso opuscolo del Vescovo Münter. La lettera colla quale me lo accompagnò è tanto di vecchia data, che non potè contenere cenno alcuno intorno ai frammenti di Ulfila da me speditigli a Coppenaghen. Mi da però un vivo sentore del piacere che Münter ha provato per l'opera d'Eusebio, così egli scrivendomi: "Le rendo infinite grazie per il Cronico di Eusebio. Quanto nuovo ed immortale merito si è acquistato l'instancabile Maio! E' un tesoro per la storia antica, e nessuno, in che parte di essa sia che travagli, potrà dispensarsi dal consultarlo. L'ho già fatto io in una piccola monografia della vita di S. Lucio I, papa, che fu il santo tutelare della mia cattedrale prima della riformazione, ed avrò spesso occasione di ritornare a questa ricchissima sorgente di cronografia". Se ella mai avesse lettera od altro da mandare a quel brav'uomo, me lo faccia pur tenere, che non mi mancheranno occasioni per trasmettergli ogni cosa», Epistolario del cardinale Angelo Mai, a cura di G. Cozza Luzi, Bergamo, 1883, 33-34. La lettera è attribuita, anche nell'indice del volume, a «O. Cattaneo» ma si tratta di un chiaro errore tra l'altro comprensibile dato la vicinanza delle forma maiuscola corsiva delle lettere $o$ e $g$. In generale per Mai - il grande filologo a cui Leopardi dedicò il terzo dei suoi Canti, Ad Angelo Mai: quand'ebbe trovato i libri di Cicerone della Repubblica - P. Treves, Lo studio dell'antichità classica nell'Ottocento, Milano e Napoli, 1962, 347-397, oltre alla voce di A. Carrannante, Angelo Mai, in 
sto giorno medesimo penso di rimettere al Signor Seifferheld, ${ }^{46}$ a norma del vostro desiderio. ${ }^{47}$

Tamburini non solo tornerà ancora nel carteggio tra i due dotti ma sarà, per stessa ammissione di Cattaneo, la «spinta» che lo portò, nel 1823, dopo oltre quattro anni, a tornare a scrivere a Münter rompendo quel silenzio, così tipico dei rapporti epistolari, che, quando protrattosi troppo a lungo, alimenta, proprio grazie alla vergogna che provoca, se stesso:

questa spinta mi fu data da una circostanza dipendente da una persona che vi sta molto a cuore, e che occupa meritatamente un posto così distinto nella stima vostra. Il buono e venerabile Tamburini ha composto recentemente un lungo Capitolo in terzarima nell'occasione del suo ottantesimosesto compleannus che fa maravigliare ognuno per la vigoria del pensiero che vi domina, e la freschezza quasi giovanile con cui ha trattato un metro dei più difficili tra i molti dell'italiana poesia. Siccome poi ha dipinto così al vivo se stesso in questi versi, così mi sono imaginato che sarebbe per riuscirvene gradita la lettura, e ne ho fatto fare una esatta copia, che ho l'onore di spedirvi oggi assieme agli augurj e saluti del Poeta ai quali vi aggiungo quelli di un altro degno Ecclesiastico che conoscete, e che vi stima infinitamente. Questi è il Canonico Tosi, che soleva accompagnarvi nelle vostre escursioni mentre soggiornavate in Pavia, e che oggi è nominato da Sua Maestà alla dignità di Vescovo di quella medesima città. Quando quest'ultimo seppe ch'io intendeva di scriverle, mi raccomandò caldamente di richiamarvi la Sua persona, lusingandosi ch'essa non sia del tutto cancellata dalla vostra memoria. Lo faccio dunque col più grande piacere, prontissimo ad onorare in tal modo una persona così degna, e che è in possesso nel paese nostro di una delle prime reputazioni nel ceto ecclesiastico. ${ }^{48}$

Dizionario Biografico degli Italiani, LXVII, 517-520 e alle molte annotazioni spesso critiche, fatte da Sebastiano Timpanaro nel suo straordinario La filologia di Giacomo Leopardi, Roma e Bari, 1997 (1a ed. 1955).

46 Louis Seufferheld di Francoforte, ma dal 1810 residente a Milano, fu amico di Mylius e poi di Manzoni e sposò, nel 1829 Carolina Maumary, sorella di quella Luisa, vedova di Enrico Blondel, che divenne la seconda moglie di Massimo d'Azeglio dopo la morte di Giulia Manzoni. Il volume - come dimostra la lettera a Jeremias David Reuß presentata alla nota 17 - venne inviato a Francoforte dove il fratello di Louis, Marquard, ancora gestiva la banca di famiglia.

47 Gaetano Cattaneo a Frederik Münter, Milano 24 marzo 1819, Det Kongelige Bibliotek, NKS $16982^{\circ}$, IV, 5, lettera 526.

48 Gaetano Cattaneo a Frederik Münter, Milano 25 gennaio 1823, ivi, lettera 527.

Sebbene sia difficile comprendere appieno a cosa si riferisca Cattaneo, e che cosa Münter esattamente volesse, è comunque interessante riportare uno stralcio che ben dà 
Tamburini e Tosi, di cui chiaramente Münter aveva ricambiato i saluti con partecipazione, se non con affetto, saranno ricordati anche nell'ultima missiva di Cattaneo che pur nell'imbarazzo di cui s'è detto in precedenza, o forse proprio per questo (come pare suggerire l'ultimo commento), in una evidente captatio benevolentiae, tacque della morte di Isimbardi, ma ci tenne invece a far sapere a Münter che: «pendant Son Séjour à Pavie, Sa Majesté a fait le meilleur accueil à Monsignor Tosi, qui a aussi obtenu, dit-on, des procureurs trés avantagentes pour son Diocèse. Le vieux Tamburini a été également, a ce qu'on m'a assuré, tres bien accueilli. Je vous annonce cela, car je suis certain de vous faire plaisir». ${ }^{49}$

\section{4. «DEGLI AMICI CHE TIENE IN QUESTO PAESE». MÜNTER E I LOMBARDI}

Nelle prime settimane del 1787, sulla via del ritorno in Danimarca, Münter visitò Pavia, tra il 19 e il 27 gennaio, ${ }^{50}$ e quindi, tra il 28 gennaio e il 12 febbraio, Milano. ${ }^{51}$ Giunse a Pavia con una lettera di raccomandazione di Mario Pagano, ma fattagli avere attraverso il Tommasi, ${ }^{52}$ per Domenico Alfeno Vario, all'epoca professore di diritto civile presso l'ateneo ticinese. ${ }^{53}$ Pagano presentò con queste parole il

l'idea della curiosità erudita di Münter e del genere di incombenze che questi soleva chiedere ai suoi interlocutori: «Sinora non mi capitò alcun Israelita abbastanza colto per potergli fare le domande di che mi pregaste nell'ultima vostra lettera. Se ancora non avete trovato chi abbia supplito alla mia povertà di relazioni con persone di quella credenza, scrivetemelo perché ho un mezzo opportuno di poter fare interpellare chi sarà in grado di corrispondenza, se non in tutto, almeno in parte alle vostre ricerche».

49 Gaetano Cattaneo a Frederik Münter, Milano 18 giugno 1825, ivi, lettera 528.

50 Frederik Münter, et Mindeskrift, Aus den Tagebüchern Friedrich Münters, III, 271-280.

51 Ivi, 280-293.

52 Notato in Perrone, La Loggia della Philantropia, 34. «Qui annessa ritroverai la lettera di Mario per Vayro in Pavia», Donato Tommasi a Frederik Münter, Napoli 9 dicembre 1786, Det Kongelige Bibliotek, NKS $16982^{\circ}$, XIV, 4, lettera 2539.

53 Al riguardo: M.C. Zorzoli, Domenico Alfeno Vario, in "...parlano un suon, che attenta Europa ascolta”, 87-93; I. Del Bagno, «Nisi utile est quod agimus, stulta est gloria». Le Institutiones iuris neapolitani di Domenico Alfeno Vario, Frontiera d'Europa 2, (2003), 179-220 e, soprattutto, D. Mantovani, Domenico Alfeno Vario professore di Diritto civile (1780-1789). L'immedesimazione polemica nell'antico, in Esortazioni alle storie, a cura di A. Stella e G. Lavezzi, Milano, 2001, 397-438. 
danese: «costui è un mio amico, è un altro me stesso, tali sono i legami che a lui mi stringono. Avrà forse bisogno della vostra assistenza nel veder biblioteche, nel conoscere letterati di costà. Io son sicuro che dimostrerete a lui quanta bontà avete per me». ${ }^{54}$ Appena giunto a Pavia Münter si recò immediatamente da Vario ${ }^{55}$ ma non ci volle molto perché, legatosi agli ambienti pavesi, si svincolasse dalla compagnia di quest'ultimo tanto che il nome del giurista salernitano, all'epoca già caduto in disgrazia presso molti colleghi e inviso a gran parte del corpo accademico, compare solo di rado nelle pagine del diario redatte durante la permanenza pavese di Münter. ${ }^{56}$

I giorni pavesi e quelli milanesi (nel capoluogo lombardo Münter venne spesso raggiunto da amici conosciuti a Pavia), emergono dalle pagine del diario $^{57}$ come un periodo intenso, ma lieto, ricco di conversazioni, di discussioni, di incontri, di visite a istituzioni culturali e a biblioteche (come a Brera e in Ambrosiana a Milano) ${ }^{58}$ ma dove ci fu anche il tempo per giocare a biliardo, come Münter annota nei suoi quaderni (la sera del 4 febbraio, a casa di Isimbardi, in compagnia di Bossi) ${ }^{59}$ o per andare a teatro. Nico Perrone, partendo dal linguaggio

${ }^{54}$ Lettera edita in R. Brienza, Martirologio della Lucania, Potenza, 1882, 52. Cito da Perrone, La Loggia della Philantropia, 35.

55 Frederik Münter, et Mindeskrift, Aus den Tagebüchern Friedrich Münters, III, 272.

56 Münter lo rivide il 20 e il 25 gennaio; ivi, III, 273 e 278. Di Vario Münter mantenne comunque un giudizio positivo, pur tacendone i difetti: «Vayro ist ein ganzer Napolitaner. gut, ehrlich, aufrichtig im höchsten Grade; aber auch ein bitterer u. unversönlicher Feind, der seine Zunge schlechterdings nicht bändigen kann, aber auch zu edel ist, um s. Feind böses zu thun, das mit s. Beleidigung nicht in Verhältniss ist», ivi, III, 278-279.

57 L'unico altro documento in nostro possesso al riguardo è la lettera a Stefano Borgia inviata da Pavia il 25 gennaio 1787 e pubblicata in Il giansenismo in Italia. Piccola antologia di fonti, a cura di P. Stella, Bari, 1972, 94-98. Ringrazio Giuseppina Basile della Biblioteca Comunale «Isidoro Chirulli» di Martina Franca per avermi fatto avere copia di queste pagine. Solo poche righe sono dedicate comunque a Pavia, dove Münter annota l'accoglienza ricevuta : «qui in Pavia sono stato sette giorni. Doveva partire oggi, e non ho trovato l'occasione, così che partirò domani colla punta del giorno. Sono stato ottimamente accolto da Zola, Tamburini ed Alpruni», Münter menziona poi di aver fatto la conoscenza «di un certo canonico Volta» e narra della vicenda di Lazzaro Spallanzani contro cui era «stato fatta la calonniosa accusa di aver rubato il museo di storia naturale di Pavia, fondato nutrito e governato da lui» e del relativo processo che ne seguì, ivi, 96-97.

58 Frederik Münter, et Mindeskrift, Aus den Tagebüchern Friedrich Münters, III, 290.

59 Ibidem. 
impiegato dalla già citata lettera di Mario Pagano, ha suggerito una affiliazione massonica per Vario: $:^{60}$ il dato, a mia conoscenza, è ancora incerto, sicuramente però gli incontri avuti da Münter - che, per usare una espressione di Croce che ha avuto in seguito grande fortuna, era in Italia in veste di «agente massonico» ${ }^{61}$ - si caratterizzarono anche per questo aspetto. Münter ebbe infatti ampi e proficui contatti con Gian Rinaldo Carli ${ }^{62}$ con Angelo Vecchi ${ }^{63}$ con il conte Wilzeck, gran maestro della loggia milanese La concordia, e vicino agli illuminati di Baviera, e quindi con Giambattista Biffi, gran maestro della loggia San Paolo la Celeste di Cremona, poi L'Aurore de la Lombardie. ${ }^{64}$ Inoltre, così come emerge con particolare chiarezza anche dal carteggio con Cattaneo, durante il passaggio in Lombardia, Münter si legò in modo speciale a Isimbardi, Bossi e Rezia (il fatto che, come prima cosa, avesse chiesto conto di loro è ben indicativo del particolare affetto che Münter doveva evidentemente sentire nei loro confronti). ${ }^{65}$ Tutti e tre ben rappresentano figure di intellettuali dai sentimenti riformatori,

60 Perrone, La Loggia della Philantropia, 34.

${ }^{61}$ Croce, Federico Münter e la massoneria di Napoli, 169.

${ }^{62}$ Al riguardo: E. Apih, Gian Rinaldo Carli, in Dizionario Biografico degli Italiani, XX, 161-167 e il volume, a cura dello Stesso, La formazione culturale di Gian Rinaldo Carli, Trieste, 1973 oltre a quello di F. De Stefano, Gian Rinaldo Carli (17201795). Contributo alla storia delle origini del Risorgimento italiano, Modena, 1942. A questi lavori vanno aggiunti due studi più recenti: B. Costa, Gian Rinaldo Carli presidente del Supremo Consiglio di economia pubblica (1765-1771), Nuova Rivista Storica 76, (1993), 277-318, A. Trampus, L'illuminismo e la Nuova politica nel tardo Settecento italiano: l'uomo libero di Gian Rinaldo Carli, Rivista Storica Italiana 106, (1994), 42-114 e C. Abbona, Le Lettere Americane di Gian Rinaldo Carli tra lumi ed "ammassi di sogni", Giornale storico della letteratura italiana 628, (2000), 241-251. 367 e 375

63 Per la sua adesione massonica: Francovich, Storia della massoneria in Italia,

64 Per Biffi, anzitutto la voce in V. Lancetti, Biografia cremonese ossia Dizionario storico delle famiglie e persone per qualsivoglia titolo memorabili e chiare spettanti alla città di Cremona, 3 voll, Milano, 1819-1822, II, 348-358 quindi F. Venturi, Un amico di Beccaria e di Verri, profilo di Giambattista Biffi, Giornale storico della letteratura italiana 134, (1957), 37-76; Id., Illuministi italiani. Riformatori lombardi piemontesi e toscani, Milano e Napoli, 1958, 385-390; G. Dossena, Per il diario del Biffi, Studia Ghisleriana 3, (1968), 1-93 e Id., Giambattista Biffi, in Dizionario Biografico degli Italiani, X, 378380 oltre a E. Santoro, Contributi alla biografa del conte Gambattista Biffi, Bollettino storico cremonese 25, (1970-1971), 69-92.

${ }^{65} \mathrm{La}$ freddezza con cui Cattaneo aveva liquidato le scelte dei suoi vecchi amici italiani di «correre lo Stadio rivoluzionario» doveva aver messo sull'avviso Münter che, a differenza di Isimbardi - che non era caduto in disgrazia dopo la Restaurazione 
affiliati alla massoneria, e dai profondi interessi scientifici ed eruditi, giovani e più o meno coetanei di Münter (solo Rezia era decisamente più anziano), i quali al momento della prova rivoluzionaria si schiereranno, a viso aperto, per questa. ${ }^{66}$ Isimbardi, Bossi e Rezia fanno insomma il paio con i più cari tra gli amici italiani di Münter, quei giovani intellettuali napoletani, anch'essi massoni, che presero tutti parte attiva allo sfortunato esperimento della Repubblica Napoletana, quali Francesco Mario Pagano, Giuseppe Albanese, Ignazio Stile, Emmanuele Mastelloni, Nicola Pacifico solo per citarne alcuni. ${ }^{67}$

Oltre ai massoni Münter frequentò però anche un altro gruppo di "radicali" e cioè a dirsi i prelati giansenisti, docenti e non, legati alla facoltà teologica dell'Università di Pavia riformata, proprio in questi anni, in chiave filo-giansenista e giuseppista. ${ }^{68} \mathrm{Si} \mathrm{è} \mathrm{già} \mathrm{avuto} \mathrm{occasione}$ di menzionare Tamburini e Tosi ma Münter ebbe contatti anche con un altro giansenista pavese che, assente dal carteggio con Cattaneo, venne invece ricordato nella già rammentata missiva di Münter a Ignaz Heinrich von Wessenberg, e cioè Giuseppe Zola. ${ }^{69}$ Di questi, con cui il dotto danese ebbe «lunghe ed amichevoli conversazioni» ${ }^{70}$ si conservano sempre presso Det Kongelige Bibliotek di Copenaghen due lettere inviate a Münter di grande rilevanza per comprendere appieno i rapporti tra questi e i giansenisti lombardi. La prima, inviata da Pavia il 10 agosto $1787,{ }^{71}$ si apre con le scuse, in questo caso evidentemente non

mantenendo anzi i propri incarichi - preferì evitare ogni ulteriore riferimento ai ben più compromessi Bossi e Rezia.

66 Isimbardi - oltre che direttore della zecca di Milano, una posizione importante, impegnativa e di grande fiducia - fece poi parte della «consulta legislativa» della Repubblica Cisalpina. A. Zanoli, Sulla milizia cisalpino-italiana: cenni storico-statistici dal 1796 al 1814, 2 voll, Milano, 1845, I, 235.

67 Perrone, La Loggia della Philantropia, 165-166.

68 Al riguardo: F. Gatti, Il periodo Giansenista della Facoltà Teologica di Pavia (1733-1797), Alba, 1954 e M. Bernuzzi, La Facoltà teologica dell'Università di Pavia nel periodo delle riforme (1767-1797), Milano, 1982. Per il connesso seminario, si veda invece: V. Pedante, Il Seminario Generale di Pavia sotto Giuseppe II, in Cattolicesimo e lumi nel Settecento italiano, a cura di M. Rosa, Roma, 1981, 205-237 e X. Toscani, Il Seminario Generale di Pavia, Annali di storia pavese 18-19, (1989), 137-144.

69 Si veda per questa influente e forse oggi troppo poco studiata, figura della vita intellettuale italiana del secondo Settecento: A. Zambarieri, Giuseppe Zola, in “...parlano un suon, che attenta Europa ascolta", 337-343.

70 Francovich, Storia della Massoneria in Italia, 427.

71 Giuseppe Zola a Frederik. Münter, Pavia 10 agosto 1787, Det Kongelige Bibliotek, NKS $16982^{\circ}$, XVI, 11, lettera 3095. 
retoriche, per il proprio ritardo; «ho tardato alquanto a scrivervi per aspettare che foste di più fermo a Copenaghen dove ora v'indirizzo le nuove lettere». ${ }^{72}$ Zola lo informa poi che «la Contessa Isimbardi mi ha commesso due tomi del fu Monsignor Litta ${ }^{73}$ che vi spedirò con alcuni libri miei e del Conte Isimbardi» e ci dà prova, ancora una volta, dello speciale rapporto di simpatia che si era dovuto creare a Milano tra Münter e Maddalena Beccaria Isimbardi ${ }^{74}$ della quale, come si è già avuto modo di vedere, chiese immediatamente conto a Cattaneo non appena ne ricevette la prima missiva temendone, purtroppo a ragione, la morte («la di lui Madre è infatti morta da varj anni, come Ella aveva avuto presentimento»). La lettera di Zola prosegue poi aggiornando Münter tanto delle sue attività che degli eventi relativi al celebre sinodo di Pistoia:

Ora si sta stampando il iv. tomo de' miei Commentarii, ${ }^{75}$ e un compendio, ch'io ho fatto ad uso del popolo e de' giovani del bel trattato di Palmieri sulle indulgenze ${ }^{76}$ dove vedrete, con quanta sincerità si parli degli abusi delle superstizioni e delle opinioni ridicole onde con vero scandalo de' buoni si è tanto osservata questa materia. Forse sarà proibito a Roma; ma non importa, abbiamo di mezzo il Po', che quando qua' fulmini scendo[n] bruti e irragio-

72 Ibidem

73 «L. Litta del Diritto di stabilire impedimenti dirimenti il Matrimonio, e di dispensarne, 1-2, Pavia 1783», Bibliotheca Münteriana, 406, n. 6413-6414.

74 Münter si trovò molto spesso a casa Isimbardi e il nome della contessa - sorella di Cesare Beccaria - ritorna più volte nelle pagine milanesi del diario dell'ecclesiastico danese (Frederik Münter, et Mindeskrift, Aus den Tagebüchern Friedrich Münters, III, 287, 288, 290 e 293). A riprova del legame che dovette instaurarsi fra i due sappiamo anche di una lettera che la nobildonna scrisse a Münter e che questi ricevette il $25 \mathrm{feb}$ braio 1787, a Chambéry, ivi, 301-302.

75 G. Zola, Commentariorum de rebus christianis Prolegomena. Adiecta de Histor. ecclesiast. fontibus et praecipuis scriptoribus mantissa, Pavia, 1787. Münter possedeva però una versione precedente di questo testo, «J. Zolae, comment. de rebus christianis prologomena, Ticini 1779», Bibliotheca Münteriana, 359, n. 5224.

76 G. Palmieri, Trattato storico-dogmatico-critico delle indulgenze, Pistoia, 1786. Mentre il volume a cui fa riferimento Zola è il Compendio del Trattato-storico dogmatico-critico delle indulgenze: con un breve catechismo sulle medesime secondo la vera dottrina della Chiesa proposto dal vescovo di Colle a' suoi parochi per servirsene d'istruzione ai loro popoli, Pavia, 1789. Per la notevole figura di Palmieri la recente scheda di Marco Bernuzzi in "...parlano un suon, che attenta Europa ascolta", 366-368 oltre a S. Cipriani, Vincenzo Palmieri, giansenista ligure, Piacenza, 1949 e G. Signorotto, La questione delle indulgenze nel Settecento italiano, Rivista di storia e letteratura religiosa 17, (1981), 49-63. 
nevoli ha la mirabil virtù di ammorzargli nelle sue acque. Dopo stamperò il v. tomo dei miei Commentarii, ${ }^{77}$ le prelazioni sopra Arnaldo, ${ }^{78}$ ed altre simili operette. Ma voi sapete quanto sia occupato; e poco tempo mi resta da stampare $[\ldots]$ vi manderò gli atti del Sinodo di Pistoia, questi si stampano anche in latino, ${ }^{79}$ e dell'Assemblea di Firenze tosto che sieno pubblicati, con alcuni anedoti che ora faccio copiare. Sempre più rimarrete persuaso della dottrina e rettitudine del Gran Duca, di Monsignor Ricci, e di altri pochissimi; come della ignoranza e della cabala di tutti gli altri. Il Gran Duca nel congedargli dall'Assemblea gli ha ripresi di questi vizii solennemente. Uno poi di quegli anedoti si è la sedizione concitata in Prato contra Monsignor Ricci per aver abolita la superstiziosa processione di una cintura che dicevasi della madonna scesa dal Cielo e taumaturga. Intanto che il buon Vescovo trovavasi all'assemblea Roma e i frati hanno eccitato questo fuoco. Si è gettata a terra la cattedra Vescovile; si sono cacciati dall'Accademia Ecclesiastica que' buoni Chierici e Sacerdoti. Si è fatta una notturna processione la più scandalosa. Il gran Duca ha represso esemplarmente questo livore; e que' sediziosi altri sono stati condannati in esiglio, altri in prigionia, altri in castello. Fra' quali il fratello di Monsignor Martini. Nostro Signor Duca egli stesso colle proprie mani ha levato quella cintura e l'ha portata a Firenze. ${ }^{80}$

77 Ritengo che Zola si riferisca al secondo tomo dei suoi Commentariorum de rebus christianis ante Constantinum Magnum, uscito a Pavia nel 1793. Il primo volume era stato stampato nel $1786 \mathrm{e}$, sebbene non compaia nella lista dei libri di Münter, è presumibile che Zola gliene facesse dono scrivendogli poiché Münter lo cita nei suoi Primordia Ecclesiae Africanae, København, 1829, 28. Münter ebbe per Zola grande ammirazione da un punto di vista scientifico come appare evidente dalla già menzionata lettera a Borgia del 25 gennaio 1787, da Pavia, dove scrisse «Zola è senz'altro uno de' più grandi letterati che abbia l'Italia. In Roma vi sono taluni che lo vogliono negare, e che l'accusano del plagiato nelle sue opere. Ma io credo conoscere almeno la più parte de' più insigni letterati d'Italia e di Germania, e vorrei che in Germania vi fosse molti come Zola», Il giansenismo in Italia. Piccola antologia di fonti, 96.

78 Verosimilmente le Praelectiones Historicae. De erroribus \& controversiis circa Christi Gratiam E Predestinationem Sanctorum exortis, Pavia, 1793. Il riferimento pare ad Arnaldo Pontaco, citato, assieme con lo Scaligero, da Zola.

79 Poco prima Zola s'era sfogato per il fatto che, specialmente in Germania, comparissero con sempre più frequenza opere scientifiche non in latino: «va bene che si stampino nella lingua natia le opere di gusto, di pubblica istruzioni etc ma le opere de' dotti [...] si dovrebbero stampare in una lingua comune a tutti i dotti dell'Europa, cioè nella latina, senza mettergli nella necessità di spendere la metà de' loro giorni ad imparar tutte le lingue dell'Europa». Zola non lo dice a Münter, con un pudore che gli fa onore, ma gli atti erano stati tradotti, in parte dal sacerdote pistoiese Matteo Soldati, in parte dallo stesso Zola che fu anche il promotore, assieme a Tamburini, dell'edizione in latino. Stella, Pietro Tamburini, 183.

80 Giuseppe Zola a Frederik Münter, Pavia 10 agosto 1787, Det Kongelige Bibliotek, NKS $16982^{\circ}$, IV, 11, lettera 3095. 
La seconda missiva, inviata oltre un anno dopo ${ }^{81}$ si apre, ancora una volta, con le scuse per il grande ritardo, «troppo lungo sarebbe il descrivere le infinite noie che m'hanno frastornato tutto quest'anno, onde secondo il desiderio mio non ho potuto coltivare la corrispondenza co' miei amici. Io però v'assicuro, carissimo Münter, che anche in mezzo a questo silenzio v'amo cordialmente e ben lo sanno tanti co' quali io faccio spessissimo onoranza e dolce memoria di voi». La lettera, che si conclude con un saluto rivolto a Münter dagli altri pavesi ( «Tamburini, Lanigan, eletto Professore di Scrittura nella università, ${ }^{82}$ Rezia, Frank, ${ }^{83}$ ec. vi arcisalutano, e si ricordano della vostra dottrina e amabilità. Io v'amo e sono di cuore tutto vostro») continua con un quadro della situazione pavese ragguagliando Münter circa la pubblicazione degli atti del sinodo di Pistoia, un evento che aveva profondamente interessato Münter e di cui questi aveva provato a tenersi informato anche sulla via del ritorno in Danimarca: ${ }^{84}$

Vi ringrazio delle nuove comunicatemi, e di cuore mi congratulo che abbiate trionfato degli emoli vostri. ${ }^{85}$ Noi pure abbiamo avuto qualche disturbo per

81 Giuseppe Zola a Frederik Münter, Pavia 15 dicembre 1788, ivi, lettera 3096.

82 John Lanigan era divenuto, nel 1788, docente di ermeneutica biblica, M. Bernuzzi, Lo spazio universitario del giansenismo pavese, in Pietro Tamburini e il Giansenismo Lombardo, 299. Per Lanigan: T. Cooper, Jobn Lanigan, in Dicitonary of National Biography, 63 voll, London, 1885-1900, XXXII, 135-137.

83 Johann Peter Frank insegnò Medicina teorico-pratica e dioica medica a Pavia dal 1785 al 1795, prima di trasferirsi a Vienna. Al riguardo l'articolo di A. Malamani, L'idea di polizia medica nel pensiero e nella pratica di Johann Peter Frank, in Esortazioni alle storie, 728-744 e il profilo di L. Bonandrini, Johann Peter Frank, in "...parlano un suon, che attenta Europa ascolta", 288-295. Ancora di grande utilità risulta poi la sua autobiografia che godette di due diverse versioni italiane: Biografia del consigliere e professore Giovanni Pietro Frank, Milano, 1802 e Biografia di Johann Peter Frank scritta da se stesso fino all'anno 1801, Pisa, 1822.

84 Come a Salisburgo il 17 aprile 1787, Frederik Münter, et Mindeskrift, Aus den Tagebüchern Friedrich Münters, III, 373.

85 Münter doveva aver comunicato a Zola la propria elezione a professore di teologica presso l'Università di Copenaghen avvenuta nel 1788 (nel 1790 diverrà poi professore ordinario e negli anni 1796-97 e 1802-1803 sarà pure magnifico rettore). L'elezione a professore straordinario non era stata però semplice, Münter era stato bensì molto contrastato e di queste difficoltà aveva fatto partecipi anche gli amici napoletani (Perrone, La Loggia della Philantropia, 20-23). Non a caso le missive di Zola sono indirizzate, per quanto riguarda la prima, a «Monsieur M.r Frideric Münter a Coppenague en Dannemark», mentre la seconda «A Monsieur M.r Frideric Münter Prof.r en Theologie dans l'Université de Copenhague en Dannemark». 
parte degli Oblati, ch'io riguardo quai piccoli Gesuiti di questo Stato. In somma costoro hanno tentato di mettere in iscompiglio la Facoltà Teologica, e di mettere il fuoco della discordia nella Università ma non è loro riuscito [...] i giovani seminaristi hanno preso un gusto grandissimo a' buoni studi; ma è incredibile la persecuzione che loro si fa da questi poco illuminati Vescovi, e dalle lor Curie. [...] Finalmente il Gran Duca di Toscana dopo la prudente dilazione di due anni ha fatto pubblicare il sinodo di Pistoia in quattro volumi in $4^{\circ}$ e gli Atti dell'Assemblea de' Vescovi della Toscana in sette gran volumi in $4^{\circ}$ magnificamente stampati nel Palazzo Pitti. Grande belle memorie vi si trovano per togliere gli abusi e le superstizioni! Grande scandalo che quattro soli vescovi sieno stati concordi nel promuovere il bene che intendea il Gran Duca, il quale e in questa e in altre occasioni si è mostrato per così dire Vescovo più zelante e illuminato ch'essi non sono. Roma è in allarme.

Le lettere di Zola si rivelano particolarmente degne di nota per quello che riferiscono a Münter circa il sinodo di Pistoia. Sebbene si sia andato sottolineando sempre di più in sede d'analisi storica il ruolo che vi ebbe Pietro Leopoldo, ${ }^{86}$ il sinodo, di cui fu promotore Scipione de' Ricci (1741-1810) e che vide Tamburini come coordinatore dei lavori, venne percepito come la più significativa esperienza del giansenismo italiano e i suoi atti dovevano suscitare in Münter grande interesse; se ne ha un riscontro nel fatto che scrivendo, nel maggio del 1788, all'Amaduzzi, un altro filogiansenista, ${ }^{87}$ ne chiedeva conto e, come vedremo, dichiarava di aspettare «ansiosamente» proprio i futuri atti. Qui, dopo aver parlato con l'amico romano ${ }^{88} \mathrm{di}$

86 C. Fantappiè, Riforme ecclesiastiche e resistenze sociali. La sperimentazione istituzionale nella diocesi di Prato alla fine dell'antico regime, Bologna, 1986; Id., Per una rilettura del sinodo di Pistoia del 1786, Cristianesimo nella storia 8, (1988), 541-562 e P. Hersche, Zum zweibundersten Jahrestag der Synode von Pisoia (1786), Internationale Kirchliche Zeitschrift 78, (1988), 243-251.

87 M. Trincia Caffiero, Cultura e religione nel Settecento italiano: Giovanni Cristofano Amaduzzi e Scipione de' Ricci, Rivista di storia della Chiesa in Italia 28, (1974), 94-126 e 30, (1976), 405-437.

88 Münter e Amaduzzi avevano stretto amicizia a Roma grazie alla comune frequentazione di Borgia, che Amaduzzi ben conosceva. Nel 1770 Amaduzzi divenne infatti sopraintendente alla Stamperia di Propaganda Fide, esattamente lo stesso anno in cui Stefano Borgia venne fatto segretario della medesima Congregazione. I rapporti tra i due andarono col tempo guastandosi ma la collaborazione, nei primi anni, fu molto proficua. Al riguardo: M. Caffiero, Le Efemeridi letterarie di Roma (1772-1798). Reti intellettuali, evoluzione professionale e apprendistato politico, in Dall'erudizione alla politica, giornali, giornalisti ed editori a Roma tra il XVII e XX secolo, a cura della Stessa e di G. Monsagrati, Milano, 1997, 68. Per Borgia e il suo ruolo nella Congregazione invece: 
novità editoriali e dei suoi Fragmenta patrum græcorum ${ }^{89}$ (di cui avrebbe voluto fare avere al più presto una copia a Stefano Borgia), scrive:

Sarà più ricca l'Italia. la prego di darmene parte; e di farmi sapere quel che potrà sapersi del Concilio Fiorentino, e del Sinodo di Pistoia de' quali ansiosamente aspetto li atti. Ora che sono in paese di libertà, non è più delitto il dichiararsi amico ed ammiratore di Monsignore di Pistoia, di Tamburini, di Zola, \& credo essere il dovere di ogni uomo da bene, di che setta tra Cristiani che sia, di far commun'affare contro i Gesuiti con tutti i buoni. Lei scrivendo a Monsignor di Pistoia, ad i suoi amici di Pavia, nominatamente al Padre Alpruni ${ }^{90}$ mi faccia il favore di assicurargli del mio perpetuo ed inviolabile attaccamento. ${ }^{91}$

Come si è già avuto modo di accennare, le lettere qui esaminate permettono di farsi un'idea più chiara dei rapporti di Münter con i giansenisti pavesi, e sono una fondamentale conferma della profondità delle relazioni che le pagine dei quaderni italiani descrivono ma cui, data l'estemporaneità delle annotazioni diaristiche, si poteva essere tentati, almeno sino ad oggi, di non dare completamente credito. Münter dovette stringere relazioni in particolare con i professori della facoltà teologica, quali Zola, Tamburini, ma anche Alpruni, così come con due della facoltà di medicina, Rezia e Frank, di cui ci sono note le convinzioni gianseniste. ${ }^{92}$ In fine, oltre ai docenti, Münter si legò pure con giovani di belle speranze (quali Bossi, Tosi ma anche John Lanigan, che non viene citato nel diario) in un ambiente in cui, al di là della retorica contemporanea, ma come appare chiaro anche dalle

J. Metzler, Ein Mann mit neuen Ideen: Sekretär und Präfekt Stefano Borgia (1731-1804), in Sacrae Congregationis de Propaganda Fide Memoria Rerum. 350 anni al servizio delle missioni (1622-1972), a cura dello Stesso, 3 voll, Roma, Freiburg e Wien, 1971-1976, III, 119-152, e il volume di saggi: Stefano Borgia nomo dalle idee nuove, a cura di R. Langella, Velletri, 2006.

89 F. Münter, Fragmenta patrum græcorum, København, 1788.

90 Francesco Alpruni barnabita e docente presso la facoltà teologica pavese, di idee gianseniste, in seguito all'invasione francese fu tra i più convinti sostenitori del nuovo regime. Al riguardo: A. Zambarbieri, Giansenismo e Rivoluzione. Francesco Alpruni, Annali di storia pavese 20, (1991), 123-146 e Id., Francesco Alpruni, in "...parlano un suon, che attenta Europa ascolta", 356-365.

91 Frederik Münter a Giovanni Cristofano Amaduzzi, Copenaghen 13 maggio 1788, Frederik Münter, et Mindeskrift. Briefwechsel Friedrich Münters, V, 10.

92 G. Natali, Il Settecento, Milano, 1973, 22. 
parole di Zola, le idee gianseniste avevano serie difficoltà ad attecchire tra gli studenti. ${ }^{93}$

Come si ha prova dai carteggi sinora esaminati Münter si affezionò sinceramente tanto a Zola quanto a Tamburini (dei quali, nella lettera ad Amaduzzi, si definisce «amico ed ammiratore») e non è un caso che, nello scrivere a Cattaneo, Tamburini, a cui Cattaneo aveva recapitato una lettera di Münter per il prelato, l'anziano professore vi aveva scorto, ma forse lo stesso Münter lo dichiarava esplicitamente, proprio dell'affetto ( $\ll i$ frattanto prego Lei vivamente di recare a quel dottissimo Vescovo i miei più vivi ringraziamenti per l'affetto, ch'egli si degna di conservarmi») che è ulteriormente confermato, se ce ne fosse bisogno, dalle continue richieste che Münter fece a Cattaneo per essere informato riguardo alla salute dell'anziano Tamburini. Che i sentimenti fossero ricambiati è confermato, oltre che dalle dichiarazioni di Tamburini e di Tosi raccolte da Cattaneo, dalla prima lettera di Zola dove l'immagine degli amici che «arcisalutano» non ha perso, anche a distanza di oltre due secoli, la sua freschezza e sincerità. Di fronte a tali dichiarazioni di bene e di amicizia può forse stupire il fatto che i contatti epistolari con il gruppo lombardo non vennero mantenuti. La cosa diviene meno sorprendente se la si confronta con quanto avvenuto con gli amici napoletani e siciliani, che con Münter ebbero un rapporto di grande intensità e un carteggio che era oramai consueto e abituale da ben prima che il dotto danese lasciasse l'Italia. Anche in questo caso non si conservano rapporti epistolari successivi al 1789, con le sole eccezioni di quelli con Francesco Mario Pagano, giunto sino al 1792 e con Melchiorre Delfico che si spinse, questo davvero in modo anomalo, sino al $1820^{94}$ ma il cui carteggio conosce, e non a caso, uno iato nella corrispondenza tra il 1790 e il $1810 .{ }^{95}$ Sono due gli aspetti che vanno tenuti presente per comprendere appieno cosa probabilmente successe. Da una parte fu lo stesso viaggio di Münter - pieno di tappe, di soste, di repentini cambi di destinazione - a rendere difficile il mantenimento dei contatti, al punto che Zola, nella prima lettera che

93 C. Capra, Aspetti delle riforme ecclesiastiche nella Lombardia austriaca, in Pietro Tamburini e il giansenismo lombardo, 39. Più in generale sulla situazione del clero lombardo in questi anni: X. Toscani, Il clero lombardo dall'Ancien régime alla Restaurazione, Bologna, 1979.

94 Perrone, La Loggia della Philantropia, 12-13.

95 Det Kongelige Bibliotek, NKS $16982^{\circ}$, IV, 10, lettere 615-625. 
abbiamo analizzato, aveva dato ragione a Münter del ritardo nello scrivergli con la precisa scelta di attendere che questi fosse «di più fermo a Copenaghen ${ }^{96}$ così da essere sicuro dell'indirizzo. O, ancora, la distanza di Copenaghen e la scarsa corrispondenza che di solito vi veniva inviata dall'Italia aggiungeva il problema, non secondario, della «via» attraverso cui far giungere le lettere, affidandole cioè a quali mani e facendogli fare quale percorso; non a caso, sempre Zola, nella seconda delle sue lettere, dopo aver comunicato a Münter di avere diversi libri con sé che intendeva spedirgli al più presto, esclamò «ma di grazia scrivetemi s'io debbo indirizzarvi questo involcro per la via indicatami l'anno scorso, oppure se debbo tenere altra strada». ${ }^{97}$ D'altro canto però saranno anzitutto gli eventi europei - prima la Rivoluzione, che investirà anche l'Italia, e poi, in secondo luogo, le guerre napoleoniche - a favorire di fatto lo sfilacciamento, e quindi la perdita, dei contatti. Münter non si dimenticò però degli amici lombardi ed è indicativo che in una lettera del 1798 Henri Grégoire, vescovo costituzionale di Blois e, secondo una celebre definizione di Francesco Ruffini, «capo assoluto di tutto il Giansenismo francese e italiano», informava il dotto danese circa gli amici italiani, citando proprio Tamburini e Zola ma menzionando, fra l'altro, anche de' Ricci, Tosi e Degola. ${ }^{99}$ La lettera di Grégoire è la prima in nostro possesso di un carteggio altrimenti ampio ${ }^{100}$ ed è quindi difficile capirne il retroterra. Tra gli appunti di Münter oggi a Copenaghen è stato possibile ritrovare la bozza di una missiva non datata che è con tutta probabilità una minuta di quella a cui il giansenista francese rispondeva nell'ottobre del 1798. Münter scriveva al giansenista francese: 3095.

96 Giuseppe Zola a Frederik Münter, Pavia 10 agosto 1787, ivi, XVI, 11, lettera

97 Giuseppe Zola a Frederik Münter, Pavia 10 agosto 1787, ivi, lettera 3096.

98 F. Ruffini, Il "masso" del Natale manzoniano e il giansenismo, in Studi sul giansenismo, Firenze, 1974, 15. Come introduzione a una figura importantissima, su cui si è scritto molto; G. Hourdin, L'abbé Grégoire, évêque et démocrate, Paris, 1989; R. Hermon-Belot, L'Abbé Grégoire et la République des savants, Paris, 2001; A. Goldstein Sepinewall, The Abbé Grégoire and the French Revolution: The Making of Modern Universalism, Berkley, Los Angeles e London, 2005 e J. Dubray, La pensée de l'abbé Grégoire: despotisme et liberté, Oxford, 2008.

99 Henri Grégoire a Frederik Münter, Parigi 15 ottobre 1798, Frederik Münter, et Mindeskrift. Briefwechsel Friedrich Münters, V, 318.

${ }^{100}$ Det Kongelige Bibliotek, NKS $16982^{\circ}$, VI, 6-10, lettere 1002-1063. 
j'ai joué dans Vos Rapports les noms de plusieurs de mes amis d'Italie, dont la mémoire m'est vraiment chère, surtout de Monsieur Zola et Tamburini at Alpruni et de Monsieur Ricci ancien Evêque de Pistoja [...] Vous m'obliguez infiniment Monsieur, si vous vouléz bien [...] me comuniquer les nouvelles que Vous avéz de l'état de nos amis communs d'Italie. ${ }^{101}$

La minuta sembra confermare insomma quelli che sinora erano solo dei sospetti e ciò̀ che Münter, avendo avuto presumibilmente notizia dei contatti che Grégoire ${ }^{102}$ intratteneva con i giansenisti italiani, di fronte alla propria impossibilità a mantenere questi contatti, gli scrisse chiedendogliene notizie. Si può notare inoltre, se ci fosse ancora bisogno di conferme della profondità di tali rapporti, come, parlando di questo gruppo di amici italiani, Münter non abbia difficoltà a definirne la memoria «vraiment chère».

\section{MÜNTER, TAMBurini E I GIANSENISTI}

Münter risultò legato a Zola e a Tamburini non solo da rapporti di simpatia umana ma anche da un evidente e profondo interesse per le posizioni religiose e politiche dei giansenisti italiani e di Tamburini in particolare. Quando lo incontrò a Pavia nel gennaio 1787 Münter conosceva infatti da tempo il pensiero del teologo bresciano e ne aveva seguito con cura anche la vicenda umana; ${ }^{103}$ già dal 1785 infatti Münter, che era entra-

${ }^{101}$ Frederik Münter a Henri Grégoire, [Copenaghen 1798], ivi, XVII, 4, lettera 44.

${ }^{102}$ I contatti di Grégoire con i giansenisti italiani sono ben noti e sono stati oggetto di diversi studi. Lasciando da parte Degola, che divenne un vero e proprio intimo del vescovo costituzionale ma che ci interessa di meno dato che Münter non lo conobbe in Italia, né lo citò mai in altra occasione, il prelato francese ebbe un importante rapporto, declinato in un ricco epistolario, anzitutto con Scipione de' Ricci (M. Vaussard, Correspondance Scipione De’ Ricci - Henri Grégoire (1796-1807), Firenze e Paris, 1963). Grégoire conosceva e apprezzava però anche molti altri. A Tamburini che venne fra l'altro citato assieme a Zola, e ad altri pavesi quali Giudici, Palmieri e Gaslini, durante il concilio nazionale del luglio 1801 (H. Grégoire, Discours pour l'ouverture du Concile national de France. Prononcé le 29 Juin 1801 (10 Messidor an 9) en l'Eglise métropolitaine de Paris, Paris, s.d., 10-11) - Grégoire dedicò addirittura la sua De la littérature des nègres (Paris, 1808, x). In generale su questo tema e per maggiori riferimenti bibliografici, si veda D. Armando, Fra riforma della Chiesa e rivoluzione: Henri Grégoire e l'Italia, Laboratorio dell'ISPF 6, (2009), 37-55.

${ }^{103}$ Come dimostra per esempio l'annotazione a diario per il 5 agosto 1786, mentre si trovava a Roma, «nach Tisch besuchte ich Orengo. Man trägt sich in Rom mit der 
to in contatto con i circoli filo-giansenisti toscani, legandosi a Scipione de' Ricci, ${ }^{104}$ aveva avuto modo di leggere durante i suoi giorni fiorentini tanto La Vera Idea della Santa Sede quanto la Analisi del libro delle prescrizioni di Tertulliano di Tamburini. ${ }^{105}$ Münter - e va tenuto ben presente - ebbe fra l'altro la ventura di frequentare i giansenisti toscani e pavesi nel momento storico che li vide più vicini e cioè quel periodo tra il 1786 e il 1789; ${ }^{106}$ agli occhi del religioso danese, come vedremo, i due gruppi parvero come un tutt'uno. A Pavia, comunque, Münter non perderà l'occasione per coinvolgere Tamburini anche in una lunga discussione teologica sul primato del vescovo di Roma che li vedrà, di fatto, sulle stesse posizioni. ${ }^{107}$ Ancora più che gli aspetti dottrinali, le ragioni di una vicinanza e di un interesse di Münter verso i giansenisti, e viceversa, furono però quelle di una sostanziale comunanza di intenti. Da una parte merita d'essere messo in evidenza come Münter condividesse con i giansenisti la feroce opposizione ai gesuiti ${ }^{108}$ ma è soprattutto l'approccio verso le «superstizioni», dalla schiavitù delle quali era necessario liberare il popo-

Neuigkeit, der Pabst habe dem Bischof von Pavia befolen, Tamburini u. Zola zu excommuniciren, dieser habe dem Keiser geschrieben, was er wolle, welcher geantwortet, ihm gelte es alles gleich»; Frederik Münter, et Mindeskrift, Aus den Tagebüchern Friedrich Münters, III, 198. Credo che possa essere utile far notare, per inciso, come l'Orengo qui nominato sia con ogni verosimiglianza lo stesso citato in una lettera di Gaetano Carrascal a Münter del 5 agosto 1788 e che Perrone (La Loggia della Philantropia, 93) dà per ignoto e che è invece da identificare con Girolamo Orengo, ecclesiastico ligure con alcuni interessi di antiquaria, dal 1804 alla morte, nel 1812, vescovo di Ventimiglia di cui si conservano a Copenaghen tre lettere, tutte inviate da Roma e datate 3 ottobre 1786 e 14 ottobre 1786 e 13 [sic] 1787; Det Kongelige Bibliotek, NKS $16982^{\circ}$, X, 16, lettere 1931-1933. L'ultima di queste, originariamente indirizzata a Pavia, presenta questo indirizzo cancellato e sostituito con Milano, segno che dovette giungervi quando Münter se ne era già andato ed è così da datarsi 13 gennaio. Si ha conferma dell'identità dei due Orengo dal fatto che nella terza lettera l'autore riferisce a Münter «scrivendo Martedì a Carrascal».

${ }^{104}$ Per i rapporti con de' Ricci: Francovich, Storia della Massoneria in Italia, 396.

${ }^{105} \mathrm{Il}$ nome di Tamburini e la lettura delle sue opere ritorna per tutto il periodo che va dal 29 gennaio al 20 febbraio 1785, Frederik Münter, et Mindeskrift, Aus den Tagebüchern Friedrich Münters, II, 221-252.

${ }^{106}$ Stella, Pietro Tamburini, 182.

${ }^{107} \mathrm{La}$ discussione avvenne il 23 gennaio, Frederik Münter, et Mindeskrift, Aus den Tagebüchern Friedrich Münters, III, 276. Per la visione del papato dei giansenisti lombardi: P. Vismara, L'anti-infaillibilisme des jansénistes lombards à la fin du XVIII siècle, in Le pontife et l'erreur: anti-infaillibilisme catholique et romanité ecclésiale aux temps posttridentins (XVII $-X X^{e}$ siècles), a cura di S. De Franceschi, Lyon, 2010, 77-104.

${ }^{108}$ Perrone, La Loggia della Philantropia, 43. 
lo cristiano, a rappresentare il legame più sentito e rilevante. Münter, è un aspetto di cui si è già detto ma su cui è forse necessario tornare, apparteneva infatti agli illuminati di Baviera, una massoneria «razionalistica», in aperto contrasto con «quella misterica della tradizione». ${ }^{109} \mathrm{E}$ ' interessante notare inoltre come il racconto, trionfale, fatto da Zola a Münter per la soppressione, a Prato, della «superstiziosa processione di una cintura che dicevasi della madonna scesa dal Cielo e taumaturga», trova il suo paio nelle lettere che gli amici illuminati napoletani inviarono a Münter per informarlo dell'abolizione, per volontà regia, del tributo della chinea, e della relativa processione durante le celebrazioni della festa dei Santissimi Pietro e Paolo. ${ }^{110}$ Inoltre, così come Zola si preoccupava di scrivere un compendio «ad uso del popolo e de' giovani» e vedeva gli atti del sinodo di Pistoia come un modo «per togliere gli abusi e le superstizioni» e per «promuovere il bene», così Donato Tommasi, riferendosi agli eventi napoletani, aveva affermato che «il tutto ha servito a spargere le più grandi verità nel volgo». ${ }^{111}$ Come risulta chiaro anche da questi due stralci, Münter e gli illuminati da una parte, e i giansenisti dall'altra, condivisero inoltre la speranza, che in entrambi andrà delusa, di un intervento attivo dei sovrani nel processo di vera e propria liberazione delle coscienze e degli spiriti. La figura di Luigi Bossi, studente vicino al giansenismo in un momento in cui pochi lo erano, e assieme membro degli illuminati di Baviera è probabilmente il migliore esempio della prossimità di idee, o di finalità, che caratterizzò i due gruppi. Tornando a Münter e a Tamburini nello specifico si può aggiungere, a motivare un comune orizzonte di prospettive e di approcci, come il giansenismo italiano - caso unico nel cattolicesimo moderno - abbia spartito con il luteranesimo tedesco e scandinavo l'idea di una centralità dell'università come luogo deputato par excellence, per non dire l'unico, alla discussione teologica e, ancora più significativo, alla definizione di ciò che si può dire "ortodossia". Per ragioni forse diverse poi, Münter e Tamburini condivisero anche un approccio irenico; se ne è visto un esempio quando Münter, scrivendo ad Amaduzzi, s'era espresso parlando «di che setta tra Cristiani che sia», mentre Tamburini, che non stava scrivendo direttamente a Münter ma che parlava di questi a Cattaneo, non dimostrò difficoltà a chiamare il

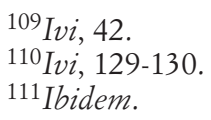


primo «vescovo», pur appartenendo Münter a una chiesa che aveva perso, sin dal 1536, la successione apostolica e sarebbe stato invece, per usare un linguaggio proprio della diplomazia cattolica in partibus infidelium, al massimo, uno «pseudovescovo».12

E' d'altro canto più difficile verificare se gli scrittori giansenisti in generale, e Tamburini nello specifico, abbiano avuto un qualche influenza su Münter. Un'analisi della biblioteca dell'ecclesiastico danese, o almeno di quella parte che è sopravvissuta sino a noi ${ }^{113}$ mostra, ed è un dato di per se stesso indicativo, una notevole presenza di testi di autori giansenisti o legati alla polemica giansenista. Vi compaiono infatti gli atti del sinodo di Pistoia ${ }^{114}$ - Zola mantenne evidentemente la parola data - assieme a due opere di confutazione di questo quali la Epistola, in qua confutantur errores, quibus abundat synodus de Ricci ${ }^{115}$ e la Damnatio del «concilio» emanata da Pio VI. ${ }^{116}$ Scipione de' Ricci è poi ben rappresentato nella biblioteca con due volumi, L'istruzione pastorale e le Lettere Pastorali, ${ }^{117}$ a cui vanno aggiunti anche una traduzione tedesca, ${ }^{118} \mathrm{e}$ forse diversi altri «opuscoli» in entrambe le lingue. ${ }^{119}$ Non di de' Ricci ma indicativamente dedicato proprio a questi ${ }^{120}$ è quindi lo scritto del lombardo Luigi Stanislao Litta, ex gesuita vicino

${ }^{112}$ Se non un vero e proprio irenismo, per lo meno un approccio irenico, per quanto riguarda Tamburini è stato notato in G. Cantarutti, Illuminismo, protestantesimo e transfert culturale fra Italia e "Germania". Tre assi di rilevazione, in Illuminismo e Protestantesimo, a cura della Stessa e di S. Ferrari, Milano, 2010, 115 e in Stella, Pietro Tamburini, 166.

${ }^{113}$ Vedi la nota 44.

114 «Acta \& Decreta Synodi Diocesanae Pistoriensis 1786. P. 1-2. Ticini 1789. (1 Vol.)», Bibliotheca Münteriana, 397, n. 6176.

115 «Epistola, in qua confutantur errores, quibus abundat synodus de Ricci, episc. Pistoriens. Ex ital. s. 1. 1789», ivi, 397, n. 6177.

116 «Pii VI. Damnatio quam plurium præpositionum excerptarum ex libro: “Atti, e decreti del consilio di Postoja 1786." Roma 1794», ivi, 111, n. 1617.

117 «Lettere pastorali di Scipione de' Ricci, vescovo di Pistoia e Prato. T. i. Firenze 1783», ivi, 397, n. 6178 e «Istruzione pastorale di Ms. Vescovo di Pistoja e Prato. Pistoja 1784 (acc. var. opuscula ejusd. argum. sem. ital.)», ivi, n. 6180.

118 «Hirtenbrief des Bischofes zu Pistoja u. Prato. Aus d. Ital. Salz. 1783 (acc. III opuscula ejusd. arg.)», ivi, n. 6179 .

${ }^{119}$ Vedi le due note precedenti.

${ }^{120} \mathrm{La}$ dedica venne inserita nella seconda edizione stampatasi a Pavia nel 1783 che è quella che, come dimostra anche il catalogo della biblioteca di Münter, questi possedette; a ulteriore riprova si può far notare come questa seconda edizione è l'unica, tra quelle che si ebbero, ad apparire in due volumi. 
agli ambienti giansenisti, ${ }^{121}$ circa il Diritto di stabilire impedimenti dirimenti e di dispensarne, che la Contessa Isimbardi fece avere a Münter ${ }^{122}$ Nella lista dei volumi posseduti dal danese compaiono inoltre due dei testi più schierati, e fortunati, del giansenismo italiano e cioè il saggio Del cattolicesimo della Chiesa d'Utrecht, ${ }^{123}$ di cui si ebbe anche una traduzione francese, uscito anonimo ma di cui fu autore Luigi Bossi e che, apprendiamo dal diario di Münter, gliene fece dono di persona quando questi si trovava a Milano, ${ }^{124}$ e quindi il Catechismo de' Gesuiti ${ }^{125}$ pubblicato, anche questo anonimo, a Lipsia dal Degola. Vanno poi aggiunti un libro scritto a quattro mani da Zola e Tamburini, ma uscito a nome del loro allievo Thaddäus Trauttmansdorff, De tolerantia ecclesiastica et civili, dato alle stampe nel 1783, in seguito all'editto di tolleranza di Giuseppe II del 1781, ${ }^{126}$ e un testo di Zola, ma pubblicato anonimo, circa la fondazione del collegio Germanico-Ungarico a Pavia, istituzione della quale, dal 1782 al 1794, Zola fu reggente e Tamburini prefetto agli studi. ${ }^{127}$ Si è deciso di non entrare qui nel tema dei rapporti di Münter con il giansenismo francese, che meriterebbe, per ampiezza e complessità, una trattazione

${ }^{121}$ Non mancarono comunque voci critiche verso quest'opera in ambiente giansenista, come quelle di Natali e di Giudici, al riguardo si veda: P. Vismara, Pietro Tamburini e il "Caso Moladori" e la questione del matrimonio nel Settecento lombardo, in Settecento religioso in Lombardia, Milano, 1994, 258. Si noti però il fatto che Zola non biasimi l'opera.

122 «la Contessa Isimbardi mi ha commesso due tomi del fu Monsignor Litta», Giuseppe Zola a Frederik Münter, Pavia 10 agosto 1787, Det Kongelige Bibliotek, NKS $16982^{\circ}$, IV, 11, lettera 3095.

123 «Del cattolicesimo della chiesa d'Utrecht e delle altre chiese d'Olanda. Milano 1786», Bibliotheca Münteriana, 468, n. 8133.

${ }^{124}$ Il 4 febbraio a Milano, Frederik Münter, et Mindeskrift, Aus den Tagebüchern Friedrich Münters, III, 289.

125 «Catechismo de' Gesuiti esposto ed. illustr. In conferenze storico-teologicomorali. ult. Ed. Lips. 1820», Bibliotheca Münteriana, 381, n. 5792.

${ }^{126}$ «Thaddei de Trautmansdorf de tolerantia ecclesiastica \& civili. Ticini 1783», ivi, 425, n. 6860. Per questo testo: Stella, Pietro Tamburini, 174-175, D. Menozzi, La patente di Tolleranza in Italia (1768-1790), Miteilungen der Österreichischen Staatsarchivs 35, (1982), 57-84 e M. Bazzoli, Il pensiero politico dell'assolutismo illuminato, Firenze, 1986, 518-524.

127 «Instituta Collegii Germanici \& Hungarici quod Josephus II, juventuti ecclesiasticæ Ticini paravit anno 1782. Ticini 1784», Bibliotheca Münteriana, 171, n. 302. Al riguardo G. Guderzo, Giuseppe II e il Collegio Germanico-Ungarico, in Studi in onore di Mario Abate, Torino, 1986, 513-533. 
a sé stante, in altra sede, ${ }^{128}$ va comunque rilevato come Münter possedesse la Lettera sulle indulgenza, versione italiana di un testo di Josse Le Plat, stampatasi a Genova nel $1798 .{ }^{129}$ E' significativo far notare quindi la presenza dello Jus ecclesiasticum universum ${ }^{130}$ di Zeger Bernhard van Espen, una delle fonti principali del pensiero tamburiniano. ${ }^{131}$ La data di pubblicazione dell'edizione di van Espen posseduta da Münter, 1791, non è priva di rilevanza e merita d'essere sottolineata; van Espen è un autore che non pare infatti essere stato conosciuto da Münter prima del viaggio italiano ma a cui questi si volse solo dopo essere entrato in contatto con i giansenisti dell'Italia centro-settentrionale. Dei libri sopra elencanti, diversi - il testo di Le Plat, il Catechismo de' Gesuiti, la condanna del sinodo di Pio VI e l'Epistola anti-ricciana - non possono essere frutto del viaggio italiano di Münter, essendo stati tutti dati alle stampe solo in seguito al suo rientro in Danimarca, e dimostrano invece il perdurare pluridecennale (il testo del Degola è del 1820) dell'interesse del religioso danese verso le posizioni teoriche dei giansenisti italiani, oltre che dei loro detrattori. Nella collezione, ragguardevole, di testi giansenisti c'è però un'assenza che spicca; gli unici scritti di Tamburini presenti sono infatti i due volumi che Cattaneo inviò a Münter nel 1819. ${ }^{132} \mathrm{E}$ ' difficile dire se vennero lasciati volontariamente, se si persero nel lungo viaggio, o, ancora, se qualcuno che s'era ripromesso di spedirglieli a Copenaghen poi non aveva voluto o potuto farlo: quello che è certo però è che Münter non ebbe con sé, una volta in Danimarca, i libri di Tamburini che sappiamo aver letto già nei giorni fiorentini del 1785 . Questo fatto, per quanto

${ }^{128} \mathrm{E}^{\prime}$ da sottolineare per lo meno, pur en passant, come il saggio di Münter sull'Inquisizione venne tradotto in francese da Henri Grégoire. Al riguardo: V. Russi Sciuti, Abolir l'inquisition d'Espagne: une lettre de l'abbé Grégoire, Annales historiques de la Révolution française 333, (2003), 121-132.

${ }^{129}$ Lettera sulle indulgenze, estratta dall'opera di Le Plat intitolata "Lettres d'un théologien-canoniste au sujet de la Bulle Auctorem fidei du 28. aout 1794". Ed e la Lettera 11. tradotta dal francese, che serve di supplemento al Trattato Storico Dogmatico sulle indulgenze, Genova, 1798; «Lettera sulle indulgenze. Genova 1798», Bibliotheca Münteriana, 406, n. 6423.

130 «Z.B. van Espen Jusecclesiasticum universum. Magunt 1791», ivi, 113, n. 1639.

${ }^{131}$ Stella, Pietro Tamburini, 178.

$132 \ll P$. Tamburini Analisi del libro delle Prescrizioni di Tertulliano, Milano 1818», Bibliotheca Münteriana, 303, n. 3915 e «P. Tamburini vera Idea della S. Sede. Milano 1818», ivi, 372, n. 5614. 
probabilmente fortuito, risulterà determinante, ma mi riprometto di tornarci fra poco, sull'impiego che Münter poté fare dell'opera scientifica di Tamburini.

Lo stato del carteggio in uscita di Münter rende particolarmente arduo comprendere se questi abbia discusso le posizioni dei giansenisti italiani con i suoi diversi interlocutori europei. Nelle lettere che ho potuto studiare il nome di Tamburini, comunque, non compare e sembra allontanarsi la possibilità, pure molto suggestiva, di una qualche influenza di Münter sulla fortuna di Tamburini nel mondo germanico (una influenza fra l'altro di per sé molto limitata come i lavori di Peter Hersche hanno dimostrato). ${ }^{133}$ Eppure il commento che fece Tamburini alle notizie inviate da Münter, «'interessamento ch'Egli si prende delle cose mie», subito dopo aver descritto i buoni segni che vengono dalla «Alemagna Cattolica» farebbe venire il sospetto che Münter non si fosse limitato a "monitorare" quanto avveniva nel mondo cattolico tedesco ma che avesse in qualche modo sostenuto, e diffuso, le idee del teologo bresciano, anche se non è possibile affermarlo con certezza. E' indubbio invece che i contatti e la conoscenza di Münter con gli ambienti del "cattolicesimo liberale" tedesco ci furono; si è già avuto occasione di citare per esempio i rapporti diretti con Ignaz Heinrich von Wessenberg ${ }^{134}$ le cui dure posizioni anti-papali e anti-curiali sono ben note, ${ }^{135}$ è inoltre sicuro che Münter conobbe l'opera ${ }^{136}$ di Carl Theodor von Dalberg, uno dei più notevoli esponenti del febronianesi-

${ }^{133}$ P. Hersche, Der Spätjansenismus in Österreich, Wien, 1977 e Tracce del Tamburini nel mondo germanico, in Pietro Tamburini e il Giansenismo Lombardo, 205-225.

${ }^{134}$ Come si è avuto già modo di dire il carteggio con Wessenberg è tardo, andando dal 15 luglio 1821 al 30 maggio 1829: Det Kongelige Bibliotek, NKS $16982^{\circ}$, XV, 13, lettere 2884-2896.

${ }^{135}$ Tra gli altri: K. Kühner, Ignatz Heinrich Freiberr von Wessenberg und seine Zeitgenossen. Lichtgestalten aus dem Katholizismus des 19. Jahrbunderts, Heidelberg, 1897 e E. Keller, Die Konstanzer Liturgiereform unter Ignaz Heinrich von Wessenberg, Freiburg, 1965 e, per il suo rapporto con Dalberg e la sua posizione nel più ampio movimento del riformismo cattolico in Germania: M. Weitlauff, Dalberg als Bischof von Konstanz und sein Konstanzer Generalvikar Ignaz Heinrich von Wessenberg, in Carl von Dalberg. Der letzte geistliche Reichsfürst, a cura di K. Hausberger, Regensburg, 1995, $35-58$.

${ }^{136}$ Come si evince, tra le altre, dalla lettera di Andreas Rudelbach a Münter, Vienna 11 dicembre 1823, Frederik Münter, et Mindeskrift. Briefwechsel Friedrich Münters, VI, 148. Nella stessa lettera è citato, fra l'altro, anche l'allora molto giovane Johann Baptist Hirscher. 
mo tedesco, così come quella di Matthias Dannenmayer ${ }^{137}$ tra i più influenti teorici del giuseppismo austriaco. Data la dominazione Asburgo in Lombardia, e la conseguente conoscenza di quanto succedeva in Austria, pare lecito supporre che Münter avesse riferito a Tamburini anzitutto dell'opera del primo e, forse, di come la Restaurazione in Germania avesse dato per definitive diverse secolarizzazioni ecclesiastiche avvenute a cavallo tra Sette e Ottocento. La lettera a Wessenberg, a ben vedere, lascia comunque il dubbio che Münter avesse provato a diffondere le idee degli amici italiani: qui infatti i nomi di Zola e Tamburini non sono introdotti ma impiegati piuttosto per spiegare chi fosse Tosi, che Münter aveva incontrato «im lombardischen Seminario bei Zola und Tamburini»; i due sono quindi autori che Münter dava per noti da parte del suo interlocutore.

$\mathrm{Al}$ di là dei rapporti epistolari è però certo, anche se per lo più passato inosservato, che Münter provò a far conoscere Tamburini e i suoi sodali al pubblico europeo. Al rientro in patria, nel 1788, Münter pubblicò un lungo resoconto dei suoi anni italiani, Efterretninger om Begge Sicilierne, samlede paa et Reise $i$ disse Lande $i$ Aarene 1785 og $1786,{ }^{138}$ a cui fece seguito una edizione tedesca, ${ }^{139}$ con diverse modifiche, e che ebbe, per le ovvie ragioni di lingua, maggiore successo e diffusione dell'originale danese (anche l'edizione italiana sarà tradotta da questa versione). ${ }^{140}$ Qui, dopo aver parlato della Sicilia, Münter inserì un importante poscritto, ${ }^{141}$ espunto, non a caso, dalla traduzione italiana, che va sotto il titolo di Beiträge zur Kenntnis der wichtigsten italienischen Universitäten und der Kirchenreformation in einigen italienischen Staaten. ${ }^{142} \mathrm{Il}$ lungo inserto è un vero e proprio articolo di propaganda dei successi, accademici quanto politici, del giansenismo italiano

137 «M. Dannenmayr institutiones historiæ eccles. N. T. Viennæ 1788», Bibliotheca Münteriana, 360, n. 5231.

${ }^{138} \mathrm{~F}$. Münter, Efterretninger om Begge Sicilierne, samlede paa et Reise $i$ disse Lande $i$ Aarene 1785 og 1786, 2 voll, København, 1788-1790.

${ }^{139}$ Id., Nachrichten von Neapel und Sicilien: auf einer Reise in den Jabren 1785 und 1786, København, 1790.

${ }^{140} \mathrm{Id}$., Viaggio in Sicilia, Palermo, 1823, il testo vedrà una nuova edizione nel 1831.

${ }^{141}$ L'unico studioso a mia conoscenza ad aver colto l'importanza di queste pagine della versione tedesca è Giulia Cantarutti nel suo Illuminismo, protestantesimo e transfert culturale fra Italia e "Germania", 115.

${ }^{142}$ Münter, Nachrichten von Neapel und Sicilien, 559-647. 
e spero che potrà essere studiato con maggiore attenzione in altra sede. Vi si descrivono comunque le riforme in Toscana e in Lombardia; si dedica grande spazio ai risultati delle Università di Siena, Pisa e Pavia; i promotori del sinodo di Pistoia vengono definiti «aufgeklärte und vernünftige Männer», ${ }^{143}$ menti sensibili e illuminate; ${ }^{144}$ Tamburini è elogiato, fra le altre cose, per il suo approccio verso i protestanti e per le sue idee «über die wahre christliche Toleranz», ${ }^{145}$ circa la vera tolleranza cristiana e, più in generale, l'insegnamento dei giansenisti, in aperto contrasto con quello dei gesuiti, è considerato «rein und strenge», ${ }^{146}$ puro e rigoroso; infine si presentono le opere scientifiche dedicate da Tamburini ai Padri Apostolici. ${ }^{147}$ Oltre a Tamburini e Zola, e ai toscani Münter troverà tempo e spazio per tessere le lodi anche di Alpruni, ${ }^{148}$ Rezia ${ }^{149}$ e Frank. ${ }^{150}$ Senza citarli per nome un omaggio ai giansenisti tornerà poi anche in un'opera del 1798, Vermischte Beyträge zur Kirchengeschichte, una raccolta di saggi di storia della Chiesa e del Cristianesimo, in un articolo dedicato all'Inquisizione, un tema particolarmente caro a Münter, ${ }^{151}$ che va sotto il titolo di Nachrichten der Inquisition in Rom, nebst Auszügen aus ibren Regeln. ${ }^{152}$ Qui Münter, illustrando la contigua Congregazione dell'Indice, prima tratta dell'attacco che la Congregazione condusse, una decina di anni prima, contro i libri «anticuriali» provenienti, oltre che dalla Germania, da Pavia e da Pistoia,${ }^{153}$ quindi critica apertamente la bolla di condanna redatta dalla Congregazione contro il sinodo di Pistoia. ${ }^{154}$ Dopo il vero e proprio omaggio al giansenismo italiano inserito nel suo Nachrichten von Neapel und Sicilien, e i riferimenti contenuti nel Vermischte Beyträge zur

${ }^{143}$ Ivi, 593.
${ }^{144}$ Concetto che Münter ripeterò ancora; «aufgeklärten Männern», ivi, 569.
${ }^{145}$ Ibidem.
${ }^{146}$ Ivi, 570.
${ }^{147}$ Ivi, 568.
${ }^{148}$ Ivi, 567 e 571.
${ }^{149}$ Ivi, 581 e 583.
${ }^{150}$ Ivi, 581.
${ }^{151}$ Sciuti Russi, Riformismo settecentesco e Inquisizione siciliana, 112-148.
${ }^{152}$ F. Münter, Vermiscbte Beyträge zur Kirchengeschicbte, København, 1798, $152-180$

153 «Diese beschäftigte sich vor zehn Jahren größtentheils mit der Untersuchung der in Deutschland herauskommenden antikurialistischen Schriften, und der in Pistoja und Pavia gedruckten Bücher», ivi, 156-157.

${ }^{154}$ Ivi, 157. 
Kirchengeschichte, Münter non tornò più, almeno apparentemente sull'argomento. La ragione è da ascriversi però, più che a un mutare di interessi, a un cambiamento nella produzione del dotto danese; Nachrichten von Neapel und Sicilien, un volume di prosa di viaggio, rappresenta infatti un unicum in una attività che andrà sempre più indirizzandosi all'antiquaria e dove quindi l'assenza di riferimenti all'attualità teologica sarà più che giustificata. L'assenza di Tamburini dovette essere condizionata in modo determinante dal fatto che, come abbiamo visto, Münter non aveva con sé testi dell'amico e, anche a volere, non avrebbe potuto menzionarlo. Proprio tale mancanza credo motivi come mai nel Haandbog $i$ den xldste christelige Kirkes Dogmebistorie, ${ }^{155}$ un manuale di storia dei dogmi dove sono costantemente trattati alcuni degli autori più studiati da Tamburini, e cioè a dirsi Origene, Tartulliano e Giustino Martire, ${ }^{156}$ il nome di Tamburini non compare mai. Pur impossibilitato a citare l'amico però, data la natura del volume - uno scritto scientifico che non lascia spazio alla polemica, all'attualità e neppure alla controversia interconfessionale dichiarata - Münter non si esimette da richiamare, e in certo senso omaggiare, $i$ giansenisti italiani e quindi Tamburini. Parlando della partecipazione dei vescovi in solidum alla Chiesa così come intesa da Cipriano, Münter, lasciando da parte la consueta asetticità accademica, si permise di inserire la considerazione: «una affermazione, che è ancora costantemente avversata all'interno della Chiesa cattolica, specialmente dai giansenisti, contro le pretese del papa». ${ }^{157}$ Non si trattava di un riferimento casuale: proprio il brano di Cipriano era stato posto da Tamburini al centro della propria argomentazione circa i rapporti tra papa e vescovi nel suo La Vera Idea della Santa Sede. ${ }^{158}$ Münter, s'è già detto molto volte, ben conosceva il testo in questione, ma è da sottolineare come l'importanza di questo passaggio specifico fosse tale da suscitare la più aspra polemica romana;

${ }^{155} \mathrm{Id}$., Haandbog $i$ den xldste christelige Kirkes Dogmehistorie, 2 voll, København, 1801-1804.

${ }^{156} \mathrm{Al}$ riguardo: Stella, Pietro Tamburini, 162-169. Specialmente per Tertulliano: A. Quacquarelli, Il De praescriptione haereticorum di Tertulliano nella polemica giansenista di Pietro Tamburini, Bari, 1953.

157 «en Sætning, som endnu idelig gientages i den katholske Kirke, især af Jansenisterne, imod Pavens Fordringer», Münter, Haandbog $i$ den xldste christelige Kirkes Dogmehistorie, II, 501.

${ }^{158}$ Il tema è affrontato al punto sesto del capitolo due della seconda parte. P. Tamburini, Vera idea della Santa Sede, Pavia, 1787, 160-163. 
il gesuita bergamasco Giovan Vincenzo Bolgeni, ${ }^{159}$ nel suo Esame della Vera idea della Santa Sede, operetta divisa in due parti e stampata in Pavia nel 1784 (1785), criticherà infatti più volte Tamburini, precisamente su questo punto. ${ }^{160} \mathrm{Il}$ riferimento di Münter ai «giansenisti» parrebbe qui, insomma, una citazione anzitutto di Tamburini, fatta a memoria e che non potè menzionare l'ecclesiastico bresciano precisamente per ragioni di metodo scientifico.

Lo studio della misura in cui Münter sia riuscito davvero a diffondere le idee dei giansenisti italiani nell'Europa settentrionale va ben al di là dei confini di questo articolo e ci si augura che venga presto affrontato; che Münter fosse intimamente legato a Tamburini doveva essere comunque chiaro almeno al pubblico francese, come risulta evidente dal necrologio del dotto danese uscito sul secondo numero del 1830 della Revue encyclopédique. Qui dopo aver accennato alla relazione con Grégoire l'anonimo redattore afferma che Münter «entretenait une correspondance intéressante avec l'Italie, par l'intermédiaire de Mgr Capece Latro, ${ }^{161}$ ancien archevêque de Tarente, et de Tamburini, le plus célèbre des théologiens de son pays». ${ }^{162}$

${ }^{159}$ Al riguardo: R. De Felice, Giovan Vincenzo Bolgeni, in Dizionario Biografico degli Italiani, XI, 274-277 e C. Sommervogel, Bibliothèque de la Compagnie de Jésus, 11 voll, Bruxelles e Paris, 1890-1932, I, 1611-1622.

${ }^{160}$ G.V. Bolgeni, Esame della Vera idea della Santa Sede, operetta divisa in due parti e stampata in Pavia nel 1784, Roma, 1836, 30-31, 78-80 e 230.

${ }^{161} \mathrm{Si}$ tratta di Giuseppe Capecelatro, ecclesiastico di simpatie gianseniste e dai vasti interessi eruditi. Arcivescovo di Taranto, sostenne la Repubblica Napoletana del 1799 e servì sotto Gioacchino Murat prima come ministro dell'Interno e poi, quando sostituito da Giuseppe Zurlo, come direttore dei musei e degli istituti di educazione. Si conserva presso Det Kongelige Biliotek un fondo di sei lettere inviate da questi a Münter, sempre da Napoli, che vanno dal 20 luglio 1824 al 30 giugno 1829: NKS 1698 $2^{\circ}, I V, 3$, lettere 473-478. I contatti tra i due dovevano essere di più antica data, anche se non risalenti ai giorni italiani di Münter (come appare evidente dalla lettere del 25 marzo 1825, dove si legge: «pur troppo mi duole di non avervi personalmente conosciuto, ma le vostre produzioni sono sufficienti pruove di un merito eminente; non è questa la prima volta che si formano ligami di perfetta stima anche a fronte delle distanze locali», ivi, lettera 474). Fu presumibilmente Münter che, per i suoi studi sulla religione dei cartaginesi, aveva per primo approcciato il prelato come emerge da una lettera a Melchiorre Delfico, [Copenaghen] 26 novembre 1808, Frederik Münter, et Mindeskrift. Briefwechsel Friedrich Münters, V, p. 217: «Ardisco di pregala [...] di informarmi, se è vero, che pochi anni addietro, nella vicinanza di Taranto si sia trovata una gran iscrizione punica, la cui metà fosse salvata da Monsign. Arciv. di Tar. Ne ò scritto l'anno passato a Monsig. Forges d'Avanzati. devo supporre che la mia lettera gli sia giunta, come 


\title{
6. Di nuovo su CatTaneo
}

\author{
Il carteggio tra Cattaneo e Münter risulta degno di attenzione, \\ come abbiamo visto, anzitutto per quanto ci rivela circa i rapporti tra
}

il Sign. Zoega la stesso consegnata alla posta di Napoli: ma la risposta di quel Prelato si è smarrito, probabilmente nella Germania - dovendo passare per tante armate» (qui, sia detto per inciso, si ha fra l'altro conferma di quanto detto circa i problemi propri della corrispondenza tra Danimarca e Italia e cioè l'incertezza delle «vie» e i problemi legati ai tumultuosi anni europei tra fine Sette e inizio Ottocento). I legami dettati da comuni interessi scientifici vennero verosimilmente rinsaldati dalla scoperta di una vicinanza di posizioni religiose tanto che Capecelatro informerà Münter del suo desiderio di fargli avere al più presto «alcune mie pastorali dirette a distruggere la supersti[zi]ose usanze introdotte nel pubblico culto», (Napoli 24 giugno 1826, Det Kongelige Bibliotek, NKS $16982^{\circ}$, IV, 3, lettera 475) o ad esclamare «Il signor Iddio vi conservi per lunghi anni per lo bene della Patria e della Religione» (Napoli 20 luglio 1824, ivi, lettera 473). Tra i volumi di Münter si incontra però solo un testo dell'arcivescovo di Taranto: «G. Capecelatro Lettera sul Battesimo Cristiano. Napoli 1817», Bibliotheca Münteriana, 319, n. 4300. Il legame dei due dovette essere stato inoltre rinsaldato dalla conoscenza che Capecelatro fece a Napoli della sorella di Münter, la scrittrice Friederike Brun, come emerge sempre dal carteggio dove a Münter si chiede più volte il favore di salutare calorosamente la sorella (ad esempio, nella lettera del 20 luglio 1824, «La mia Amica Brun mi ha dimenticato, e prego voi di rimproverarla», o, ancora, il 30 giugno 1829, «Mille saluti alla mia cara Amica e vostra sorella», Det Kongelige Bibliotek, NKS $16982^{\circ}$, IV, 3, lettere 473 e 478). Il rapporto tra i due, certo amichevole e "prossimo", non sembrerebbe motivare, almeno a prima vista, quanto riportato nel necrologio; la cosa si spiega però, da una parte, con la reputazione di Capecelatro in Francia negli ambienti prossimi a Grégoire (P. Stella, Il giansenismo in Italia, 3 voll, Roma, 2006, II, 282; da notarsi poi che qui si adotta la formula non canonica di «ancien archevêque de Tarente» che è un calcolo di quella impiegata da Grégoire di «ancien évêque de Blois»), dall'altra, tenendo presente come, Capacelatro fu l'ultimo dei corrispondenti stabili che Münter ebbe in Italia e potrebbe aver giocato un ruolo significativo, oggi ancora non investigato dalla critica, nell'aiutare la diffusione dei risultati scientifici di Münter e delle sue opere negli ambienti meridionali e napoletani in particolar modo, ambienti questi che Münter aveva conosciuto bene ma che erano usciti falcidiati dai tragici fatti che fecero seguito alla Repubblica Napoletana. Forse non a caso la traduzione italiana degli Efterretninger om Begge Sicilierne uscì a Palermo nel 1823. Per Capacelatro, si veda: B. Croce, L'Arcivescovo di Taranto, in Uomini e cose della vecchia Italia, Bari, 1927, 158-181 e la voce di Pietro Stella, Giuseppe Capecelatro, in Dizionario Biografico degli Italiani, XVIII, 445-452 mentre per Friederike, la sorella di Münter, D. Helsted, Friederike Sophie Christiane Brun, in Dansk biografisk leksikon, II, 584-587; K. Klitgaard Povlsen, Friederike Brun around 1800: episodes from travelling in Italy - the lake of Albano, Analecta Romana Instituti Danici 28, (2001), 103-108 e Ead., Sophienholm, Lyngby-Bogen, 2000-2001.

162 B.G., Nécrologie. Danemark - Frèderic Munter, Revue encyclopédique 46, (1830), 521-522. 
Münter, Tamburini e il giansenismo lombardo, aprendo, forse, addirittura un nuovo fronte di ricerche sull'influenza di quest'ultimo nei circoli dotti dell'Europa settentrionale. Prima di concludere credo che sia necessario tornare un momento su Cattaneo e provare a valutare se le lettere che si sono analizzate sin qui possano contenere anche informazioni di qualche rilievo circa le idee del numismatico milanese. Si è già avuto occasione di segnalare, per esempio, la critica del periodo rivoluzionario che pare trasparire, senza alcuna ambiguità, dalla maniera con cui Cattaneo descrisse a Münter le parabole umane di Bossi e di Rezia e più che comprensibile se si pensa al ruolo di fedele servitore dello stato austriaco svolto da Cattaneo a Milano; le lettere gettano però un po' di luce anche sulle convinzioni religiose del numismatico lombardo, un aspetto sul quale, almeno a mia conoscenza, non si è scritto molto.

La seconda delle lettere di Cattaneo è di particolare rilevanza a questo riguardo proprio perché è lecito immaginare che questi ben poco sapesse delle idee religiose del suo interlocutore (a lui noto specialmente in quanto erudito) e non vi si poté adattare. Le considerazioni del numismatico italiano risultano così timorose, e circostanziali, ma, esattamente per questo, particolarmente rilevanti. Cattaneo, non utilizzando - oserei dire non a caso - la prima persona ma preferendo una più neutra forma impersonale, definì comunque Tamburini «uomo cotanto rispettabile, e celebre in Europa». L'affetto di Münter per Tamburini e la scoperta di una vicinanza del primo alle posizioni del secondo dovettero "sciogliere" Cattaneo che arriverà ad esprimersi in toni addirittura irriverenti nei confronti di Roma, come, per esempio, quando riferirà a Münter che Tamburini «è sempre vegeto $[\ldots]$ ad onta dei fulmini del Vaticano». ${ }^{163}$ La vicinanza di Münter a Tamburini, tuttavia, potrebbe essere la vera causa, in un tentativo di ingraziarsi il religioso danese, di quel modo di riferirsi a Tamburini come «il buon», «il buono e - addirittura - venerabile» (con un aggettivo non scevro da una precisa connotazione religiosa) e solo in ultimo, ad amicizia compromessa, «le vieux». La lettera a Rossari però, dove Tamburini è definito «incomparabile», fornisce la conferma che i sentimenti di apprezzamento nei confronti di questi da parte di Cattaneo fossero, e vadano considerati, sinceri. Se è insomma impossibile stabilire una vicinanza

${ }^{163}$ Gaetano Cattaneo a Frederik Münter, Milano 13 gennaio 1819, Det Kongelige Bibliotek, NKS $16982^{\circ}$, IV, 5, lettera 525. 
alle idee teologiche dei giansenisti pare altresì certo che ci fu in Cattaneo, così come emerge dal carteggio, un apprezzamento sincero per Tamburini. Va d'altro canto fatto notare, oltre alla semplice vicinanza a Tosi («quando quest'ultimo seppe ch'io intendeva di scriverle, mi raccomandò caldamente...»), anche una vera e propria stima nei suoi confronti («che è in possesso nel paese nostro di una delle prime reputazioni nel ceto ecclesiastico»), stima che potrebbe indicare un atteggiamento critico, se non addirittura polemico, verso un certo centralismo romano. Non è possibile a dirsi, anche se non pare inverosimile, che tale atteggiamento critico combaciò con una precisa posizione di filogiansenismo, $\mathrm{o}$, piuttosto, con la prossimità ad ambienti, come quelli del Mylius o anche di Manzoni, a cui Cattaneo era legato, e di simpatie "liberali" in senso lato.

\section{CONCLUSIONI}

La corrispondenza che si è provato ad esaminare, pur nella sua minutezza, presenta diversi spunti di interesse. Al di là del carteggio numismatico, degno di nota anzitutto per quanto riguarda gli studi imperniati attorno alla collezione di monete cufiche conservate a Milano, le lettere risultano di grande rilevanza per gettare luce sui rapporti tra Münter e i giansenisti italiani, anzitutto quelli gravitanti attorno all'Università di Pavia e specialmente Pietro Tamburini, e poi, in secondo luogo, per provare a formulare qualche ipotesi circa le posizioni religiose di Gaetano Cattaneo che sembrano, se non affini, per lo meno simpatetiche con quelle dei giansenisti. Durante i suoi giorni italiani, il danese ebbe occasione di frequentare i circoli filo-giansenisti a Roma e, soprattutto, quelli toscani, in un momento di grandi speranze e di forte unità di tutto il movimento. Sulla via del ritorno in Scandinavia Münter fece poi la conoscenza di Pietro Tamburini e del gruppo pavese con i quali strinse vere e proprie amicizie e a cui, grazie a Giuseppe Zola, provò a rimanere in contatto anche dopo il suo ritorno in patria. La vicinanza tra l'illuminato di Baviera ed ecclesiastico luterano Münter e i giansenisti fu tale che, a più riprese, il primo provò a illustrare le posizioni dei secondi, sempre in modo estremamente favorevole, al pubblico colto dell'Europa del Nord. Fu la mancanza delle opere scientifiche dei giansenisti, e di Tamburini in particolare, pur in una biblioteca che ne comprendeva forse le più significative tra 
quelle di polemica e di pubblicistica, che con tutta probabilità impedì a Münter di dare maggior peso nei propri lavori all'attività dei conoscenti italiani. In fine il carteggio risulta prezioso per studiare i rapporti di Münter con la Lombardia. Le lettere di Cattaneo offrono infatti notevoli riscontri a quella che sino ad oggi era l'unica fonte circa il passaggio lombardo del dotto danese, e cioè le pagine del diario. Le lettere aiutano a meglio comprendere con chi Münter avesse legato maggiormente, e per quali motivi, e sono una importante riprova dell'intensità di alcune amicizie - come quelle con Isimbardi, Bossi, Rezia e Zola, oltre che con Tamburini - che questi strinse durante il proprio soggiorno a Pavia e a Milano. 
